\title{
Experimental assessment of the effect of temperature gradient across an aeroacoustic liner
}

\author{
F. Méry,* E. Piot, ${ }^{\dagger}$ D. Sebbane, ${ }^{\ddagger}$ Ph. Reulet, ${ }^{\S}$ and F. Simon, ${ }^{\mathbb{T}}$ \\ ONERA/DMPE - Université de Toulouse, F-31055, Toulouse, France \\ A. Carazo Méndez $\|$ \\ Airbus Operations SAS, F-31060, Toulouse, France
}

\begin{abstract}
This study investigates the temperature effect on the impedance of conventional singledegree-of-freedom liners, both without and with grazing flow. Experiments are performed in a controlled environment, with a detailed monitoring of the temperature all along the liner sample. The liner impedance is either derived from the reflection coefficient measured in a normal impedance tube, or is educed with an inverse method from acoustic velocity or wall pressure fields measured in the ONERA grazing flow duct. The influence of the acoustic source level on the temperature of the sample is also addressed, which enlights strong multiphysics coupling between acoustics, flow and thermal phenomena.
\end{abstract}

\section{Nomenclature}

Liner perforated sheet hole diameter, $(\mathrm{mm})$

Liner perforated sheet thickness, $(\mathrm{mm})$

Liner porosity

Acoustic velocity incident to the plate, $(\mathrm{m} / \mathrm{s})$

Total height of the liner sample

Density of the mean flow, $(\mathrm{kg} / \mathrm{m} 3)$

Friction velocity

Mass flow, $(\mathrm{kg} / \mathrm{s})$

$M, M_{b} \quad$ Mach number, Bulk Mach number

$f \quad$ Frequency, $(\mathrm{Hz})$

$\omega \quad$ Pulsation

$k \quad$ Wave number

$\Phi \quad$ Acoustic phase, (rad)

ls $\quad$ Loudspeaker signal, (V)

$C \quad$ Acoustic wave complex amplitude, (Pa)

$\Omega_{o b s} \quad$ Observation region for impedance eduction

$Z=R+i X$ Acoustic impedance with $R$ the resistance and $X$ the reactance

$Z_{t} \quad$ B2A exit acoustic impedance

$\theta_{N L} \quad$ Non-linear effect resistance term

$\beta \quad$ Acoustic reflection coefficient

$\alpha \quad$ Absorption coefficient

$(x, y, z) \quad$ Axial, transverse and vertical coordinates, $(\mathrm{mm})$

$(U, W) \quad$ Axial and vertical velocity, $(\mathrm{m} / \mathrm{s})$

$(\bar{U}, \bar{W}) \quad$ Axial and vertical velocity, $(\mathrm{m} / \mathrm{s})$

$(u, w) \quad$ Axial and vertical acoustic velocity, $(\mathrm{m} / \mathrm{s})$

$P, \bar{P}, p \quad$ Pressure, Mean pressure, fluctuating pressure, $(\mathrm{Pa})$

\footnotetext{
*Research Scientist, fabien.mery@onera.fr

†Research scientist, estelle.piot@onera.fr

†Technician, delphine.sebbane@onera.fr

${ }^{\S}$ Research scientist, philippe.reulet@onera.fr

IResearch scientist, frank.simon@onera.fr

"Engineer, arnulfo.carazo-mendez@airbus.com
} 


$\begin{array}{ll}.^{*} & \text { Conjugate complex operator } \\ c_{0} & \text { Sound celerity, }(\mathrm{m} / \mathrm{s}) \\ T_{b} & \text { Bottom surface temperature of a sample }(\mathrm{K}) \\ T_{u} & \text { Upper surface temperature of a sample }(\mathrm{K}) \\ T_{a m b} & \text { Ambient temperature over the sample }(\mathrm{K}) \\ \Delta T & \text { Thermal gradient in the liner }(\mathrm{K}) \\ B i & \text { Biot Number } \\ h & \text { Heat transfer coefficient } \\ \kappa_{b o d y} & \text { Thermal conductivity coefficient }\end{array}$

\section{Introduction}

Future engines, such as UHBR/UHPE Ultra High Bypass Ratio/Ultra High Performance Engine, will be designed to maximize the propulsion efficiency (fuel burn) while keeping the engine noise as small as possible. This will be enabled through several innovations, among which a lightweight, low drag, slimline nacelle and thinner, shorter outer cowls for the fan case. Moreover, as UHBR engines will require more heat dissipation, the integration of larger and hotter heat exchangers will have important impacts on all nacelle components design, including acoustic liners. Also, this new environment has increased the interest in innovative components ensuring both thermal exchanges and acoustic absorption [1, 2]. The larger fan diameter will shift the fan noise signature towards the lower frequencies, making the aforementioned integration issues even more complex. Particularly, the effects of thermal gradients inside the liner need to be correctly modeled. The presence of a temperature gradient over a liner can also be found in the automotive industry.

Conventional liners are made of honeycomb cavities topped by a perforated face sheet; their noise damping efficiency is driven by their acoustic impedance, which is an homogenized notion used for representing the material as a wall boundary condition in noise propagation simulations. To ensure an accurate design of the noise reduction, it is therefore necessary to increase the reliability of the acoustic liners impedance models regardless the environmental conditions. For conventional perforate-over-honeycomb liners (either single-degree-of-freedem (SDOF) or double-degree-of-freedem (DDOF)), a large amount of publications address the viscous, grazing flow and non linearity effects (see for instance [3] or [4] for some recent studies on these issues). However, very little is known on the effect of temperature on the acoustic performance of conventional SDOF liners. Temperature effects have mainly been addressed for perforated walls used in gas turbine combustors or in aero-engines combustion chambers. A comprehensive review on combustor liners can be found in Lahiri \& Bake's paper [5], which shows that very few studies investigated the influence of temperature on the acoustic impedance of a liner. The effect of temperature for Helmholtz resonators is addressed in several papers for combustion issues [6] [7]. In Bies \& Wilson [8], the effect of high sound pressure level of a Helmholtz resonators with a small orifice is presented measuring the effect on the temperature inside the cavity. Few studies can be found regarding conventional SDOF liners. Lahiri et al. [9] proposed acoustic impedance measurements in combustion room conditions (high temperature and high pressure). Burd \& Eversman [10] proposed a methodology to perform impedance tube measurements on SDOF at high temperature. The classical impedance tube post-processing is associated with a finite element method taking into account the temperature profile in the tube. Elnady et al. [11] measured the acoustic properties of a single orifice-over-cavity liner placed in an oven: the temperature at the surface of the sample was higher than the temperature at the back of the cavity, with a difference up to 100K. Experiments were performed for several sound pressure levels (either in the linear or non linear regime of the acoustic response), without any mean flow. The impedance was measured with an in-situ technique. It was found that the impedance change at high temperatures could be well predicted by simply changing the properties of air (density, viscosity and speed of sound) in a semi-empirical impedance model [11]. As a statement of fact, an increase of the temperature yields a decrease of the reactance due to a change of the speed of sound inside the cavity, and a slight increase of the resistance (at least while the viscous effects are dominant in the orifice resistance, compared to the nonlinear effects). Kabral et al. [12] completed this study dealing with several liners in an impedance tube and in a grazing flow facility. The effect of high temperature is fairly well predicted but the main difficulty is to accurately determine the effect of grazing flow. Rademaker et al. [13] performed in-situ impedance measurements on a SDOF liner submitted to a hot grazing flow ; the temperature was measured at the surface and within the cavity of the liner, and appeared to be 50 to $100 \mathrm{~K}$ lower than the total temperature in the duct. However no detailed monitoring of the temperature within the liner is presented. As in Elnady et al. [11] paper, it was found that the temperature only changed the liner reactance, since the liner resistance was mainly driven by the grazing 
flow effects. One can also cite Bothien \& Wassmer paper [14], in which the impact of density change on the resonance frequency of Helmholtz resonators is addressed : the resonator and the rig are flooded by either krypton or air gas in order to mimic the change of mean density created by a hot/cold air jump. As in Elnady et al. [11] paper, it is found that a impedance model can correctly reproduce the experimental results as soon as it takes into account the change of density across the resonator neck.

This study investigates the temperature effect on the impedance of conventional SDOF liners, both without and with grazing flow and both in the linear and nonlinear regime with respect of the acoustic sound pressure level. On contrary to the papers listed previously, for most of the study the surface of the liner is kept at a temperature lower than the backing of the cavity, with a temperature gradient up to 150K. Experiments are performed in a controlled environment, with a detailed monitoring of the temperature all along the liner sample. The liner impedance is either derived from the reflection coefficient measured in a normal impedance tube, or is educed with an inverse method from acoustic velocity or wall pressure fields measured in the ONERA grazing flow duct [15]. The influence of the acoustic source level on the temperature of the sample is also addressed, which enlights strong multiphysics coupling between acoustics, flow and thermal phenomena.

\section{Description of the experiments and impedance eduction methodologies}

\section{A. Tested liners : samples definition}

The liners are conventional SDOF liners made of a resistive perforated sheet mounted onto square cavities. Several liner samples have been manufactured through an additive metallic manufacturing process, at the dimensions of either the ONERA impedance tube (cylinder of $38 \mathrm{~mm}$ diameter) or the B2A test cell (parallelepiped of $50 \mathrm{~mm}$ width and $150 \mathrm{~mm}$ length). The samples are made of EOS MaragingSteel MS1, which is a pre-alloyed ultra high strength steel in fine powder form, which has been optimized especially for processing on EOSINT M270 systems. All samples were manufactured by Airbus through additive manufacturing. Pictures of the impedance tube samples are shown in Fig 1 Channels have been designed within the samples in order to integrate thermocouples at the bottom and at the surface of the liner. A total of 4 liners have been manufactured according to the characteristics given in Tables 1 and 2 , where $\phi$ corresponds to the hole diameter, $\sigma$ the liner porosity and $\delta$ the plate thickness. These liners have been designed in order to fit with industrial requirements that are not presented here. Under grazing flow, only one liner sample has been tested (\#3). The overall dimensions of the samples are 50x50x150mm in order to fit in the B2A facility.

Table 1 General geometric characteristics of the samples

\begin{tabular}{ll}
\hline \hline Parameters & value \\
\hline Height of the inner cavities & $48 \mathrm{~mm}$ \\
Back skin thickness & $1 \mathrm{~mm}$ \\
Total height of the sample $L_{s}$ & $50 \mathrm{~mm}$ \\
Size of square cavities & $10 \mathrm{~mm}$ \\
Thickness of cavity walls & $0.5 \mathrm{~mm}$ \\
Thickness of exterior walls of the sample & $1 \mathrm{~mm}$ \\
\hline \hline
\end{tabular}

Table 2 Geometric characteristics of each liner perforated sheet

\begin{tabular}{llll}
\hline \hline Sample \# & $\phi(\mathrm{mm})$ & $\sigma$ & $\delta(\mathrm{mm})$ \\
\hline 1 & 1 & $5 \%$ & 1 \\
2 & 1.5 & $5 \%$ & 1 \\
3 & 1.5 & $15 \%$ & 1 \\
4 & 1 & $15 \%$ & 8 \\
\hline \hline
\end{tabular}

A thermal pre-design study was performed to choose the power of the heating devices, both for the impedance tube and the B2A setup. This study is based on a 1D approach for solving the heat equation within the liner sample, with 


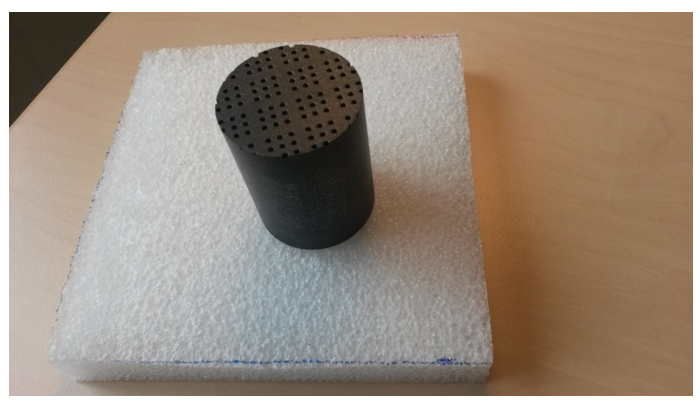

Figure 1 Liner sample example

the assumption that there is continuity of the heat flux and of the temperature between each layer of the sample. An equivalent thermal conductivity of the liner, denoted by $k_{\text {body }}$, is computed from the thermal conductivity of each layer of the liner, i.e the upper sheet (the influence of the perforations is neglected), the cavity cells (an homogenization is performed to take into account both the contribution of the air and of the cells walls) and the backing sheet. Both the upper/backing sheets and the cells walls are made of EOS MS1 material. In its powder form, the thermal conductivity of the material is $\kappa_{b o d y}=15 \mathrm{~W} / \mathrm{m} / \mathrm{K}$. A thermal test was conducted to assess the thermal conductivity on the final material, once it has been manufactured by additive manufacturing. The applied measurement technique can be found in [16]. The thermal conductivity of the material after the manufacturing is $23 \mathrm{~W} / \mathrm{m} / \mathrm{K}$.

Let $T_{b}$ and $T_{u}$ be the temperature of the bottom and the upper surface of the sample and $T_{a m b}$ be the temperature of the ambient air (in the impedance tube configuration) or of the grazing flow (in the B2A configuration). Let $h$ be the heat transfer coefficient between the sample surface and the ambient air. In the impedance tube configuration, $h$ is given by the sum of the natural convection heat transfer coefficient and the radiation heat transfer coefficient; both values are derived from empirical correlations. In the $\mathrm{B} 2 \mathrm{~A}$ configuration, $h$ is the convection heat transfer coefficient given by the Colburn's correlation [17] for a turbulent duct flow. Consider that the bottom sample temperature is controlled with a heating device of power surface density $P_{d}$, in order to maintain a given $\Delta T=T_{b}-T_{a m b}$ value. Under the 1D assumption, the following set of equations can be derived from the heat equation:

$$
\begin{aligned}
T_{u} & =T_{a m b}+\frac{P_{d}}{h}, \\
T_{b} & =T_{u}+\frac{P_{d} \times L_{s}}{k_{\text {body }}}, \\
P_{d} & =\frac{T_{b}-T_{a m b}}{1 / h+L_{s} / k_{\text {body }}},
\end{aligned}
$$

where $L_{s}$ is the thickness of the sample. The solution of these equations, and therefore the ratio between the inside and outside temperature difference $\left(T_{b}-T_{u}\right.$ vs $\left.T_{b}-T_{a m b}\right)$, is strongly linked to the value of the Biot number, defined as:

$$
B i=\frac{h \times L_{s}}{\kappa_{b o d y}} .
$$

Indeed, it can be shown that $B i=\frac{T_{b}-T_{u}}{T_{u}-T_{a m b}}$. A Biot number larger than one means therefore that there is a significant temperature gradient inside the sample, while a Biot number smaller than 0.1 implies that the heat conduction inside the body is much faster than the heat convection away from its surface, and temperature gradients are negligible inside of it. The pre-design study yields a Biot number around 0.4 for the impedance tube configuration and around 5 for the $\mathrm{B} 2 \mathrm{~A}$ configuration.The Biot number is significantly higher in the B2A duct case than in the impedance tube since in the B2A, the thermal convection is forced. A few Watts are needed as heating power in the first case, while up to 50 Watts are required in the flow duct experiments. The experimental results presented in the following sections will show that this pre-design thermal assessment is enough for pre-sizing the heating device. Nonetheless, some discrepancies are observed in terms of thermal gradient. Mainly in the impedance tube, the thermal gradient appears to be much higher than expected. The difference may come from thermal leaks through the insulating material used around the sample. 


\section{B. Description of the experimental setups}

\section{Impedance tube with thermal gradient}

The measurements are carried out in a heated impedance tube (Fig 2). This tube makes it possible to determine the acoustic impedance of a material subjected to a thermal gradient. It consists of a tube with a speaker at one end and a sample holder heated at the other end. A cooling system is located all around the tube to maintain a controlled temperature within it. Along the tube, 3 microphones are distributed for the measurement of sound pressure. Thermocouples can be placed along the tube (microphone position) and within the sample to be tested.

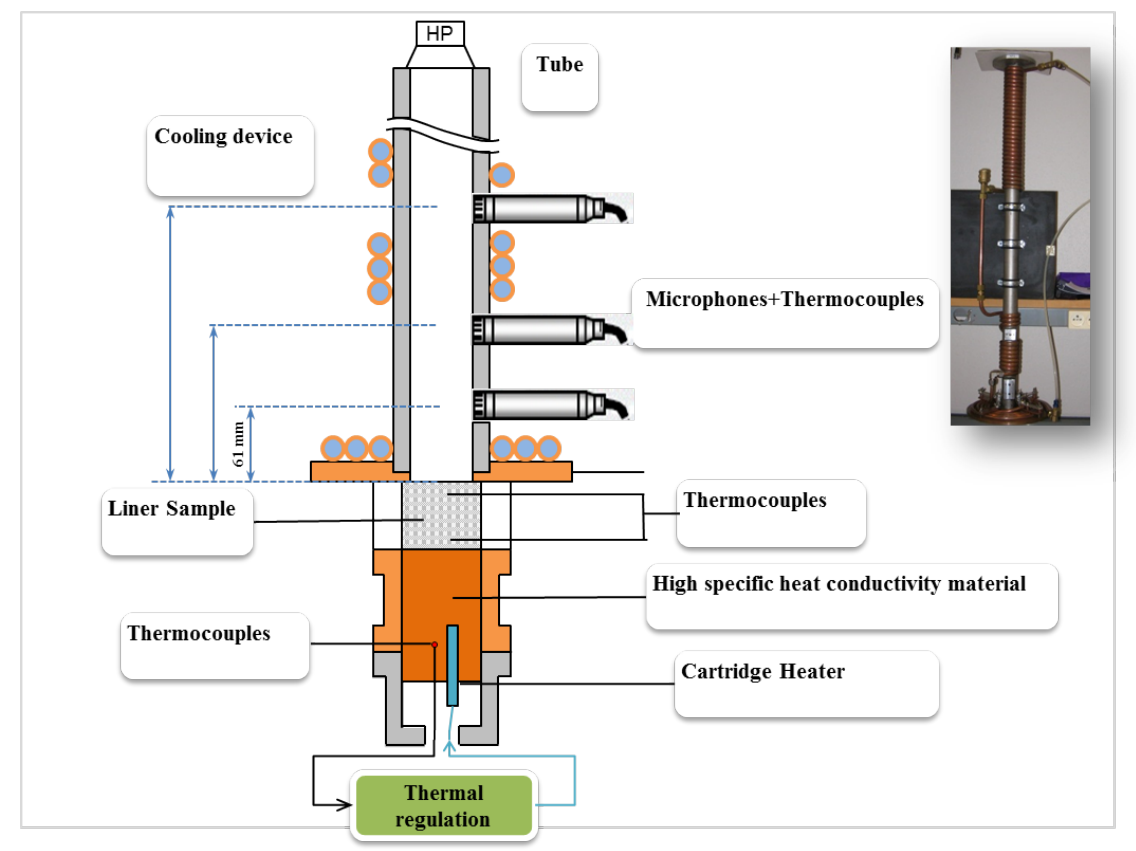

Figure 2 Impedance tube with thermal gradient

A thermal characterization of the tube with a liner sample is carried out. Two configurations are measured: (i) characterization for a thermal gradient of $70^{\circ} \mathrm{C}$ established between the bottom of the liner and the ambient air inside the tube, and (ii) characterization for a thermal gradient of $150^{\circ} \mathrm{C}$ established between the bottom of the liner and the ambient air inside the tube.

In order to reach a stationary thermal situation, the set up needs around one hour time. Fig 3 shows the results of the thermal characterization for two different thermal gradients. The cooling system makes it possible to obtain, within the tube, an homogeneous temperature at the microphone measurement position (differences less than $3^{\circ} \mathrm{C}$ ). Whatever the value of the imposed thermal gradient, approximately $60 \%$ of the temperature difference occurs within the sample and $40 \%$ occurs between the surface of the sample and the ambient air, which corresponds to a Biot number of 1.5. The sample edges are in contact with the cooled system (less than $5 \mathrm{~mm}$ ) which could explain this large difference with the thermal pre-sizing Biot number results.

\section{ONERA B2A duct with thermal gradient test section}

The aeroacoustic test bench at ONERA is made of a stainless steel tube of section $50 \mathrm{~mm} \times 50 \mathrm{~mm}$; its total length is about $4 \mathrm{~m}$. A $0.2 \mathrm{~m}$-long test section is equipped with two silica windows for optical access. The termination is equipped with a quasi-anechoic outlet, leading to an upstream reflection coefficient smaller than 0.2 for frequencies higher than $500 \mathrm{~Hz}$. A mean flow of bulk Mach number $M_{b}$ up to 0.3 can be provided. The static flow temperature can be accurately regulated, from the ambient temperature up to $300^{\circ} \mathrm{C}$. All along the study, the static flow temperature was imposed to be equal to $20^{\circ} \mathrm{C}$. In the test section, this flow shows fully developed turbulent boundary layers, with a turbulence rate of a few percent at the center of the test section. As shown in Fig. 4 , flow propagates from left to right. The test bench is connected to a pressurized air tank. A mass flow control valve can regulate the mass flow with a very high accuracy (less than $1 \%$ error on the imposed mass flow). This mass flow $\dot{m}$ is measured with the flow meter 


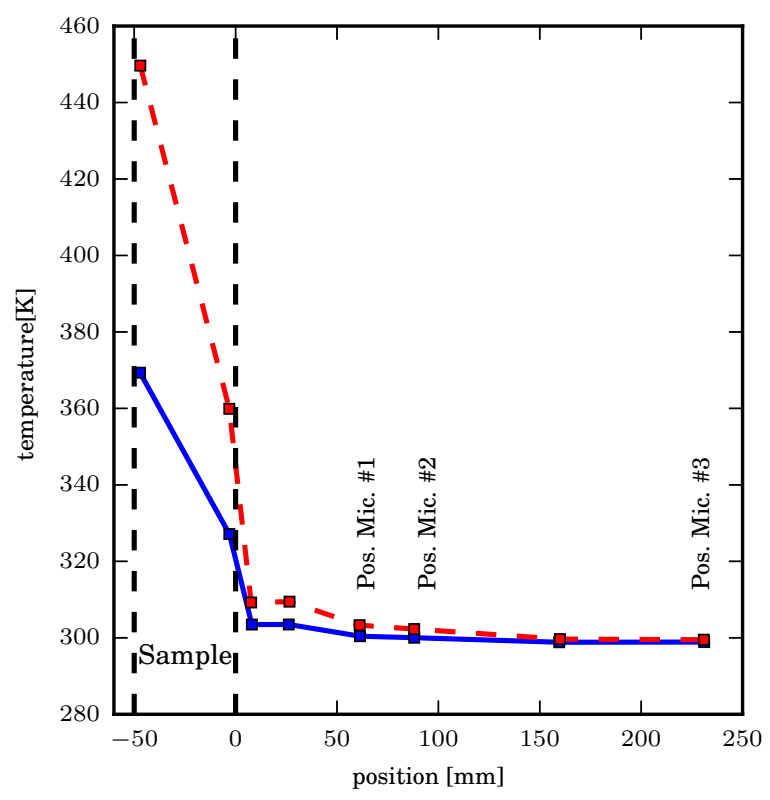

Figure 3 Thermal characterization of the impedance tube, dashed line $\Delta T=150 \mathrm{~K}$, solid line $\Delta T=70 \mathrm{~K}$

Rosemount 485 Annubar installed on the mass flow control valve. Upstream the test section, the static pressure is also measured. The bulk Mach number can be thus derived from the mass flow definition under the incompressible flow hypothesis:

$$
\dot{m}=\rho_{0} c_{0} M_{b} S,
$$

with $\rho_{0}$ the flow density derived from the static flow temperature and the static pressure upstream the test section, $c_{0}$ obtained from the static flow temperature and $S$ the surface of the test section.

Two acoustic drivers are mounted upstream of the test section, and are used to generate tones (usually multi-sine signal) at up to $140 \mathrm{~dB}$ over a frequency range of 0.3 to $3.5 \mathrm{kHz}$ (i.e., the no-flow cut-off frequency of the duct). When a multi-sine source is used, the OASPL within the duct is much higher (usually $10 \mathrm{~dB}$ more) than the SPL at each tone frequency. The surface of the test liner forms a $150 \mathrm{~mm}$-long portion of the lower wall of the flow duct. It should be noted that the liner spans all the duct width.

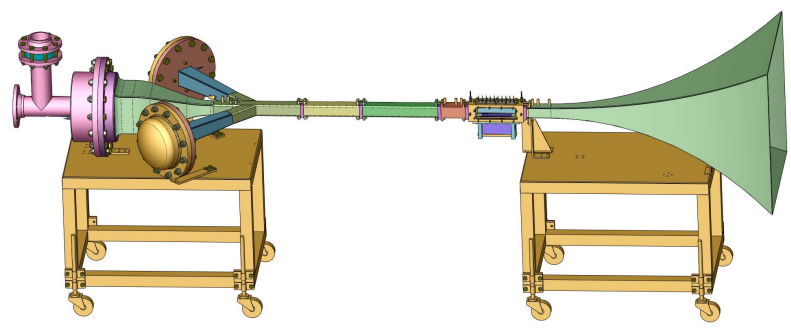

Figure 4 Sketch of B2A Aero-Thermo-Acoustic test bench.

Sixteen microphone locations are available in the upper wall (opposite the liner) to measure the acoustic pressure field from upstream of the liner leading edge to downstream of the liner trailing edge. Flush-mounted B\&K 4182 microphone probes are used (probe diameter of $1.2 \mathrm{~mm}$ ). Usually, only two upstream locations are considered to measure the amplitude of the incident acoustic plane waves[18]. However, for the pressure-based impedance eduction method, one microphone probe is moved along the whole set of microphone locations. The cross-spectrum between the microphone and driver signal is acquired, which provides a phase reference for the pressure measurements.

Fig 5 shows the new test section built for this study : a heating plate is placed under the sample to heat it. This 
heating system can reach a maximum temperature of $170^{\circ} \mathrm{C}$. In order to ensure a good thermal conductivity between the sample and the heating device a specific thermal paste is placed between the heating plate and the sample backing plate. It ensures also a good positioning of the sample. Fig 6 shows the evolution of temperature for a given bulk Mach number $\left(M_{b}=0.26\right)$ of the grazing flow in the duct, both in the flow and at the bottom of the sample. The temperature is particularly steady which ensures good condition to perform measurements during a long duration of time. Some thermocouples have been also placed inside the samples in order to assess the temperature distribution within the liner cells.

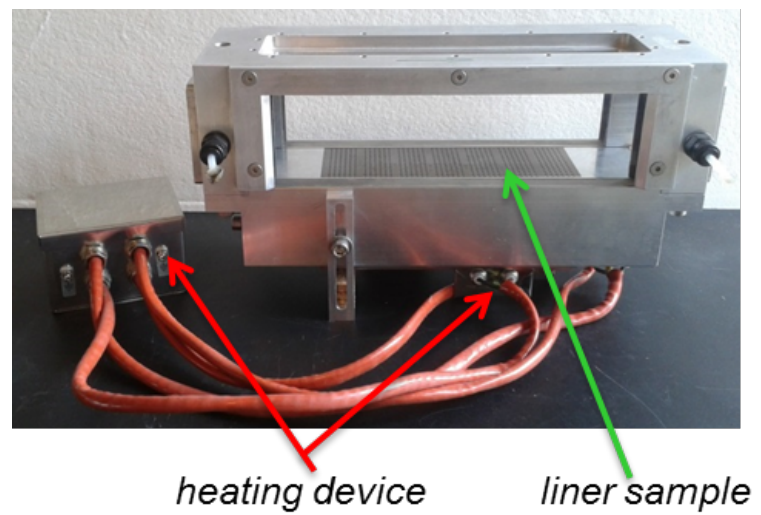

Figure 5 B2A new test section with heating system underneath the sample

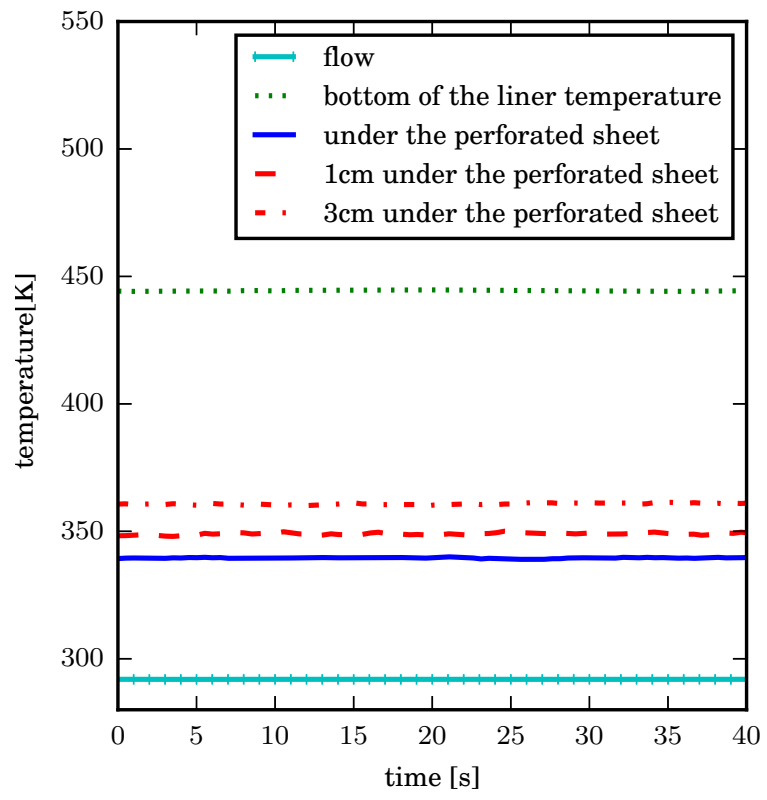

Figure 6 Evolution of temperature along time. The bulk Mach number is set to 0.26

The Biot number is around 2.1, while the pre-design was expecting a value of 5. The B2A setup thus has a lower value of Biot number than expected, but it is still in the same order of magnitude : most of the thermal gradients occur within the cavity. 


\section{Instrumentation and impedance eduction methods}

\section{Two-microphone method for measuring the incident and reflected pressure waves}

Both in the impedance tube and in the B2A tests, a two-microphone method is used to decompose the sound field in the duct into an incident and a reflected wave (see [18]). This analysis is only performed below the first cut-off frequency of the duct, so that only plane waves have to be considered. In the impedance tube configuration, this method yields directly the reflection coefficient $\beta$ of the liner sample, and therefore its impedance made dimensionless by the impedance of air. The relation between the reflection coefficient $\beta$ and the impedance $Z$ is:

$$
Z=\frac{1+\beta}{1-\beta} .
$$

This two-microphone method is also used in the B2A duct to assess the sound pressure level incident into the sample, i.e the sound pressure level of the downstream wave denoted by $A$, while the upstream reflected wave is denoted by $B$.

Two microphone probes B\&K 4182 are flush mounted to the wall, at a distance $x_{1}$ and $x_{2}$ upstream of the sample. We note $p_{1}$ and $p_{2}$ the sound pressure they measure. The spacing between the two probes is $2.8 \mathrm{~cm}$, which according to Boden \& Abom [19] minimizes errors in the [600,3450] $\mathrm{Hz}$ frequency range. In order to also minimize measurement errors at lower frequencies, the measurements are repeated by switching the two microphones and an average of the obtained frequency response function is taken: this makes it possible to overcome the intrinsic phase shift between the microphones. Using the complex notation, and by introducing the pulsation $\omega=2 \pi f$ of the considered frequency, the pressure field in the test section at a given position $x$ reads :

$$
p(x, t)=\operatorname{Re}\left(P(x) e^{i \omega t}\right)
$$

with $P(x)=A e^{-i k^{+} x}+B e^{-i k^{-} x}$ where $k^{+}$and $k^{-}$are the wave numbers and $\mathrm{A}$ and $\mathrm{B}$ are the complex amplitudes of the forward and backward waves. The $\mathrm{x}$-axis is oriented from left to right and has its origin at the liner upstream location, i.e. at the position of the impedance discontinuity. For a uniform flow of Mach number $M$, we have:

$$
k^{ \pm}= \pm \frac{\omega / c}{1 \pm M}=\frac{ \pm k}{1 \pm M},
$$

with $k=\omega / c$. The two-microphone method allows the assessment of the sound pressure level $S_{A}$ of the downstream wave (i.e. $20 \log \frac{P_{A}^{r m s}}{2.10^{-5}}$ with $P_{A}^{r m s}=\frac{|A|}{\sqrt{2}}$ ) and $S_{B}$ of the upstream wave (i.e. $20 \log \frac{P_{B}^{r m s}}{2.10^{-5}}$ with $P_{B}^{r m s}=\frac{|B|}{\sqrt{2}}$ ) from the sound pressure levels $S_{1}$ and $S_{2}$ measured at the microphones, by solving the following set of equations :

$$
\begin{aligned}
& S_{A}=S_{1}-20 \log \left(2\left|\sin \frac{k \Delta x}{1-M^{2}}\right|\right)+10 \log \left(1+2\left|H_{21}\right| \cos \left(\phi_{21}-\frac{k \Delta x}{1-M}\right)+\left|H_{21}\right|^{2}\right), \\
& S_{B}=S_{1}-20 \log \left(2\left|\sin \frac{k \Delta x}{1-M^{2}}\right|\right)+10 \log \left(1+2\left|H_{21}\right| \cos \left(\phi_{21}-\frac{k \Delta x}{1+M}\right)+\left|H_{21}\right|^{2}\right),
\end{aligned}
$$

with $\Delta x=x_{2}-x_{1}$ and $H_{21}=\left|H_{21}\right| e^{i \phi_{21}}=P_{2} / P_{1}$ the frequency response function between the microphones. Further details can be found in Leon et al. [20] or Primus et al. [21]. In the present study, $M$ is set to 0 for the impedance tube post-processing and $M$ chosen equal to $M_{b}$, the bulk Mach number, for B2A duct post-processing.

\section{Non-intrusive measurement of the acoustic velocity field}

The B2A duct is equipped with a Laser Doppler Anemometry (LDA) system, in order to measure the streamwise and vertical velocity components $u$ and $w$ of the flow. As this measurement technique is time-resolved, both the mean and fluctuating parts of the velocity can be measured. Especially, the acoustic velocity field can be obtained with a specific post-treatment technique. The acoustic velocity is defined here as the component of the signal that is correlated with the loudspeaker signal. It can be educed from the extraneous noise (mainly due to the turbulent flow) by a technique similar to the three-microphone signal enhancement technique [18]. It consists in calculating the cross-spectral density function $G_{U, l s}$ (resp. $G_{W, l s}$ ) between the velocity streamwise signal $U$ (resp. vertical velocity $W$ ) and the loudspeaker 
signal $l s$ (see [20, 22, 23]). The auto-spectral density function of the acoustic velocity reads :

$$
\begin{aligned}
G_{u} & =\frac{\left|G_{U, l s}\right|^{2}}{G_{l s}}, \\
G_{w} & =\frac{\left|G_{W, l s}\right|^{2}}{G_{l s}},
\end{aligned}
$$

where $G_{l s}$ is the auto-spectral density function of the loudspeaker signal. The acoustic velocity in the frequency domain is then given by

$$
\begin{aligned}
u & =\sqrt{G_{u}} \exp [\mathrm{j} \Phi(U / l s)], \\
w & =\sqrt{G_{w}} \exp [\mathrm{j} \Phi(W / l s)],
\end{aligned}
$$

where the phase $\Phi(. / l s)$ of the acoustic velocity, referenced by the loudspeaker, is defined as

$$
\begin{gathered}
\Phi(U / l s)=\arctan \frac{\operatorname{Im}\left(G_{U, l s}\right)}{\operatorname{Re}\left(G_{U, l s}\right)}, \\
\Phi(W / l s)=\arctan \frac{\operatorname{Im}\left(G_{W, l s}\right)}{\operatorname{Re}\left(G_{W, l s}\right)} .
\end{gathered}
$$

\section{Impedance Eduction Methodology}

In the impedance tube configuration, the impedance is directly derived from the measured reflection coefficient, as explained previously. In the B2A configuration, a more complex methodology has to be used : an impedance eduction method, which is based on the two-dimensional time-harmonic linearized Euler equations (LEE). The underlying model is briefly summarized here for convenience, but all details can be found in [21].

The linearized Euler equations, written in non-conservative form, with an $e^{i \omega t}$ time dependence, assuming homentropic flow is:

$$
\mathrm{L} \varphi=0,
$$

where

$$
\mathbf{L} \varphi=\mathrm{j} \omega \varphi+A_{x} \frac{\partial \varphi}{\partial x}+A_{z} \frac{\partial \varphi}{\partial z}+B \boldsymbol{\varphi}
$$

and

$$
\boldsymbol{A}_{x}=\left(\begin{array}{ccc}
\bar{U} & 0 & c_{0} \\
0 & \bar{U} & 0 \\
c_{0} & 0 & \bar{U}
\end{array}\right), \boldsymbol{A}_{z}=\left(\begin{array}{ccc}
\bar{W} & 0 & 0 \\
0 & \bar{W} & c_{0} \\
0 & c_{0} & \bar{W}
\end{array}\right), \boldsymbol{B}=\left(\begin{array}{ccc}
\partial_{x} \bar{U} & \partial_{z} \bar{U} & 0 \\
\partial_{x} \bar{W} & -\partial_{x} \bar{U} & 0 \\
0 & 0 & 0
\end{array}\right)
$$

Components of vector $\varphi=\left(u, w, \frac{p}{\rho_{0} c_{0}}\right)^{\top}$, where $\rho_{0}$ is the density of the mean flow, represent the acoustic perturbations around the sheared mean flow of axial and transverse velocities $\bar{U}$ and $\bar{W}$, respectively. The flow being assumed to be homentropic, the energy equation is replaced by the state equation $p=c_{0}^{2} \rho_{0}$., with $\rho_{0}$ the acoustic density.

As we consider a sheared mean flow profile, the impedance boundary condition on the lined wall is the standard one

$$
p= \pm \rho_{0} c_{0} Z w,
$$

where the sign is chosen depending on whether the upper or lower wall is considered. To avoid any singularities in the rigid wall case, the boundary condition $(19)$ is expressed as a function of the normal incidence reflection coefficient $\beta$. On the rigid walls the reflection coefficient $\beta$ is then taken as 1 .

At the source plane, the state vector $\varphi$ is taken to be equal to $C \varphi_{0}$, where $\varphi_{0}$ is the plane-wave solution in a rigid duct with uniform flow Mach number $M_{0}$, corresponding to the bulk Mach number of the actual shear flow $U(z)$. As this plane-wave solution is chosen with an arbitrary amplitude taken to be equal to 1 , the complex coefficient $C$ stands for the source amplitude. $C$ can be either set to the measured value of the acoustic pressure at the source plane, when 


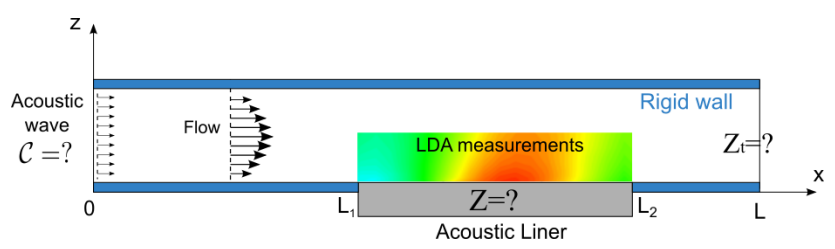

Figure 7 Impedance eduction from LDA measurements

available, or included into the eduction process to reproduce at best the measured acoustic field. At the exit plane, either a non-reflecting or an exit impedance boundary condition can be enforced. The first option is only used when the data suggests that this is proper, i.e. when no standing-wave can be observed in the downstream hard wall section. As the LDA measurement system does not extend to the exit plane, this information can only be provided by microphone measurements downstream of the lined section. As these additional measurements are not systematically performed, the exit impedance boundary condition is commonly used. This will be the case for all the computations shown in this paper. As a consequence, during the search procedure, both $C$ and $Z_{t}$ are used as design variables, in addition to $Z$.

A discontinuous Galerkin (DG) scheme is chosen to solve Eq. 177) and the associated boundary conditions. In this method, discontinuities are allowed at the interface between two elements. An example of how such a method can handle discontinuities at hard-soft wall interfaces is found elsewhere [24]. A triangular mesh of Lagrange $\mathbb{P}_{1}$ elements is used. To ensure the connection between elements and to apply the boundary conditions, a numerical flux is defined. An upwind numerical flux is used to ensure connection between interior cells, while a centered flux is chosen at the boundaries (see previous papers [21, 25] for more details).

The inverse problem is defined by the minimization of the following objective function:

$$
\mathcal{J}=\frac{\int_{\Omega_{o b s}}\left\{\left.\varphi\right|_{\mathrm{DG}}-\left.\boldsymbol{\varphi}\right|_{\text {Meas }}\right\}^{\top}\left\{\left.\varphi^{*}\right|_{\mathrm{DG}}-\left.\varphi^{*}\right|_{\text {Meas }}\right\} \mathrm{d} x \mathrm{~d} z}{\int_{\Omega_{o b s}}\left\|\left.\varphi\right|_{\text {Meas }}\right\|^{2} \mathrm{~d} x \mathrm{~d} z},
$$

where $\Omega_{o b s}$ is the observation region and .* is the conjugate complex operator. In the present study, the impedance eduction method is based on LDA measurements above the test liner (see Fig. 7), $\Omega_{o b s}$ is a rectangular $(x, z)$ region and the $(u, w)$ velocity components of the state vector are considered in function (20).

A BFGS-B (Broyden, Fletcher, Goldfarb, Shanno for bounded variables) algorithm is used to solve the optimization problem [26]. The analytical expression of the gradient of $\mathcal{J}$ with respect to each of the search parameters $Z, Z_{t}, C$ is obtained via an adjoint method, as explained in Primus et al.[21].

\section{Results and discussion}

\section{A. Impedance tube results and analysis}

The samples are tested in the impedance tube with a white noise at different Over All Sound Pressure Levels (OASPL) $116 \mathrm{~dB}, 126 \mathrm{~dB}$ and $146 \mathrm{~dB}$ measured at the first microphone in order to evaluate any impact of the SPL on the impedance values. The frequencies range from $100 \mathrm{~Hz}$ up to $5000 \mathrm{~Hz}$. In Fig 8 , the effect of the sound pressure level is presented without any temperature gradient. Samples \#1, \#2 and \#3 have a strongly non-linear behavior. Sample \#4 has a less non-linear behavior which can be explained by the thickness $(8 \mathrm{~mm})$ of the perforated sheet. 


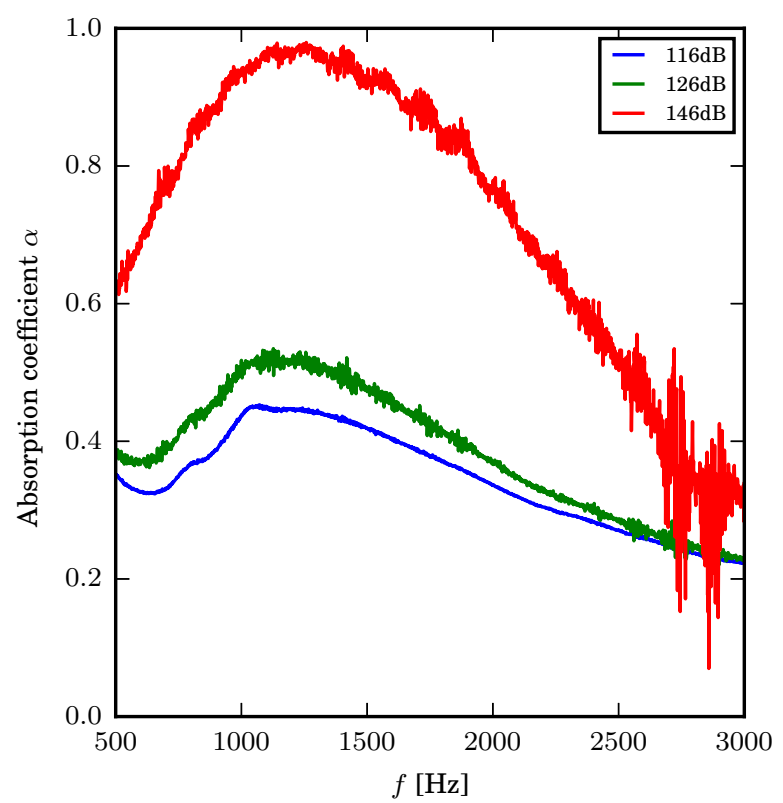

(a) sample \#1

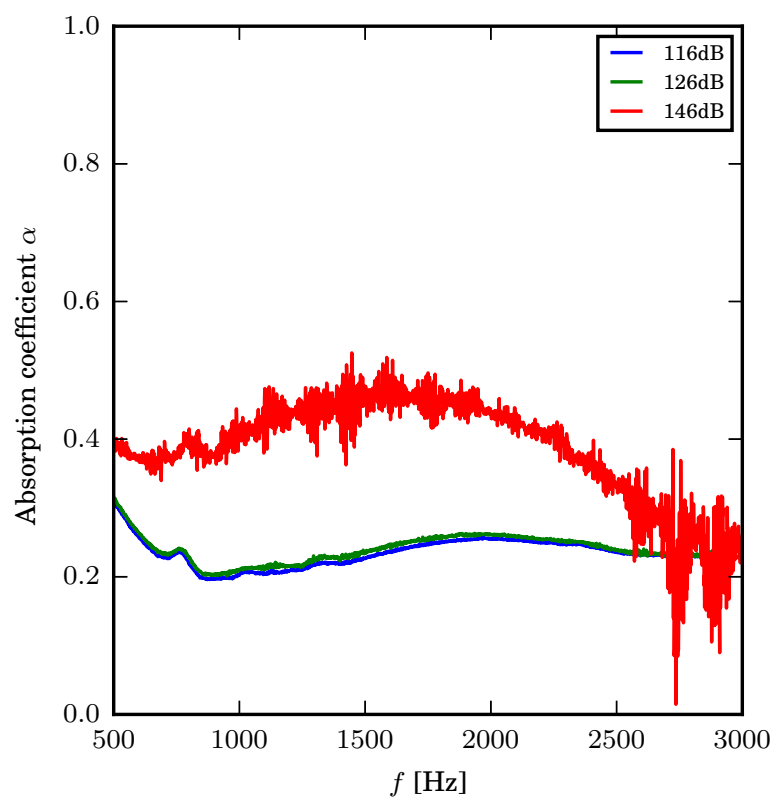

(c) sample \#3

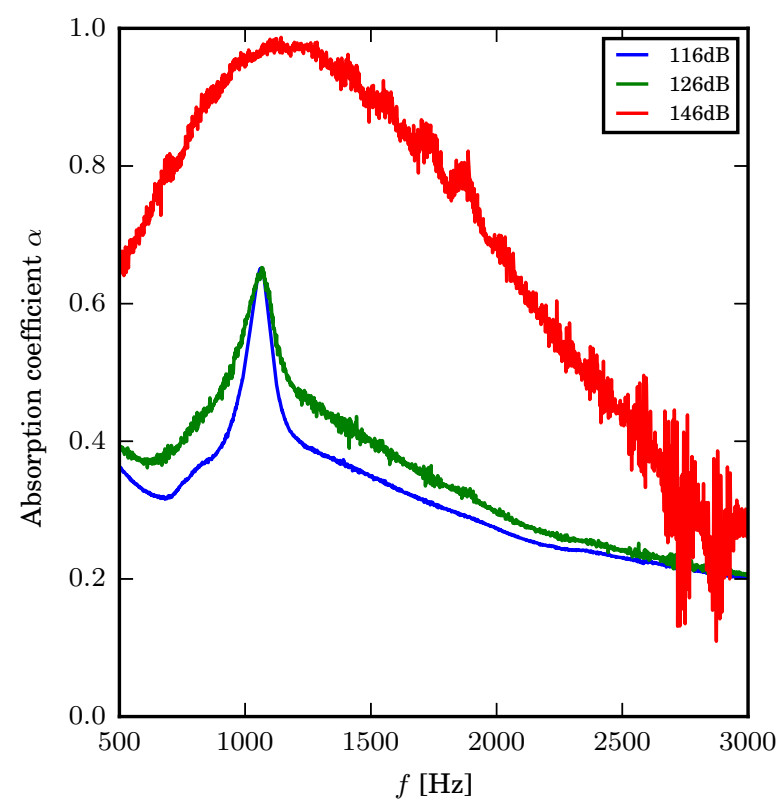

(b) sample \#2

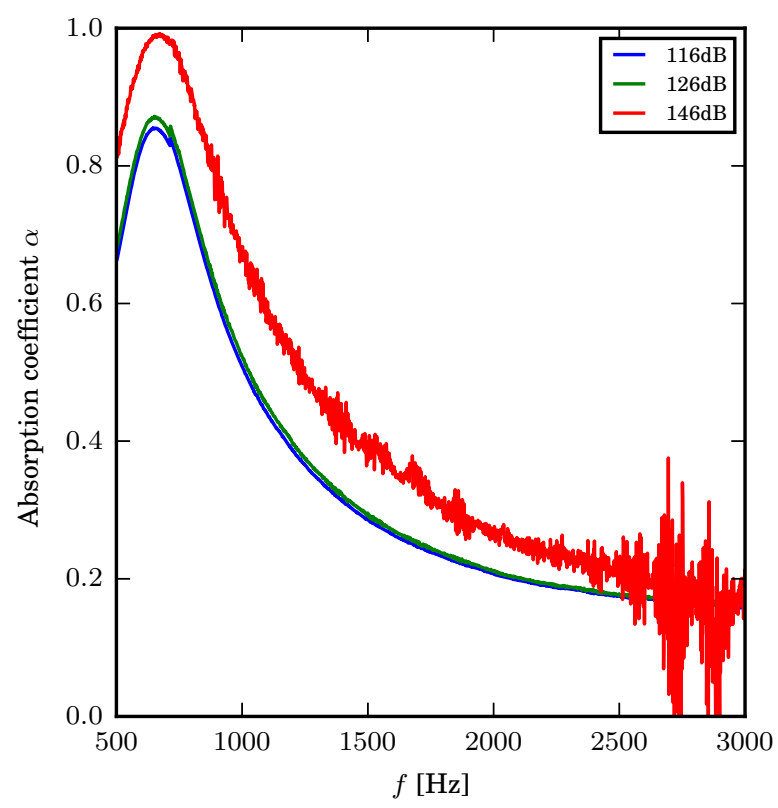

(d) sample \#4

Figure 8 OASPL level effect on absorption coefficient on samples \#1, \#2, \#3 and \#4 


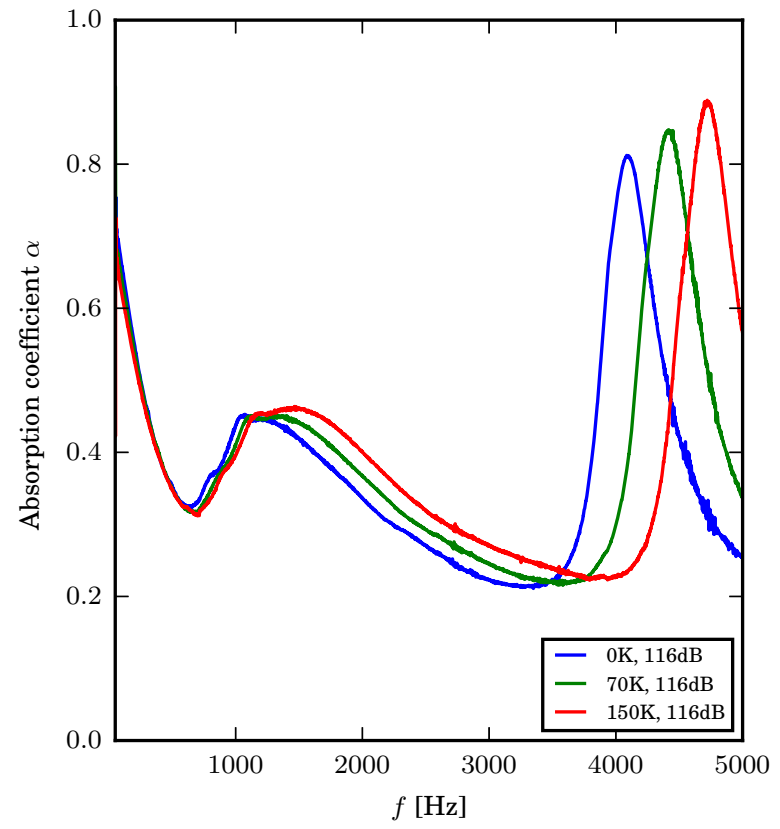

(a) Absorption

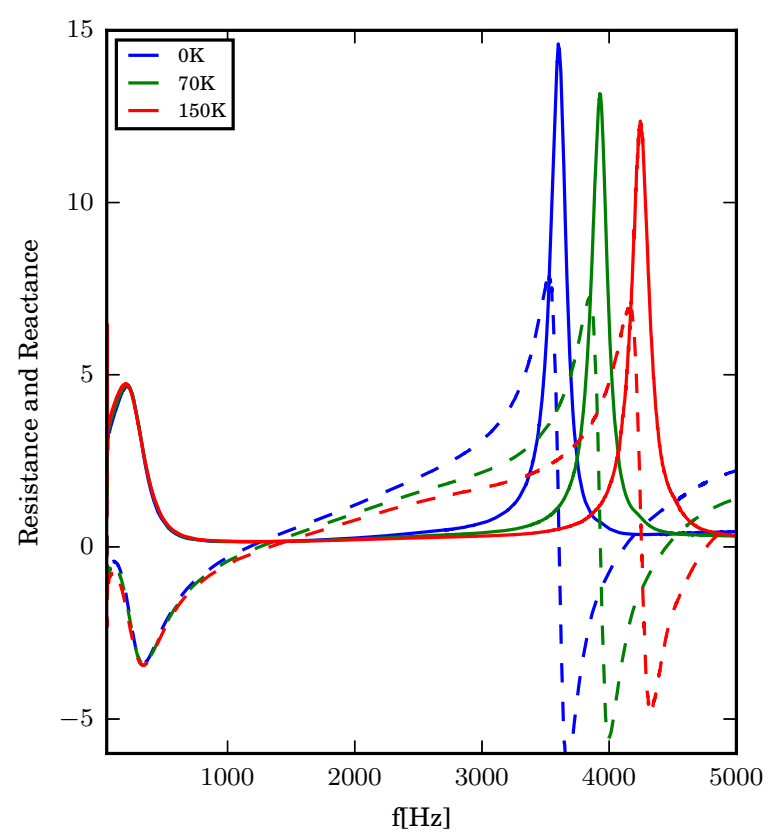

(b) Impedance : resistance line, reactance dashed lines

Figure 9 Thermal gradient effect on impedance and absorption for sample \#1 for $\Delta T=0 \mathrm{~K}, \Delta T=70 \mathrm{~K}$ and $\Delta T=150 K, \mathbf{O A S P L}=116 \mathrm{~dB}$

Figure 9 shows the effect of the thermal gradient on the absorption coefficient and the impedance for sample \#1. The main phenomenon observed is a shift to a higher frequency of the liner anti-resonance : the anti-resonance occurs at higher frequencies due to the temperature effect. These modifications on the resistance and reactance explain the modifications of the absorption coefficient and the shift on maximum absorption in the application frequency range (up to $3 \mathrm{kHz})$. 


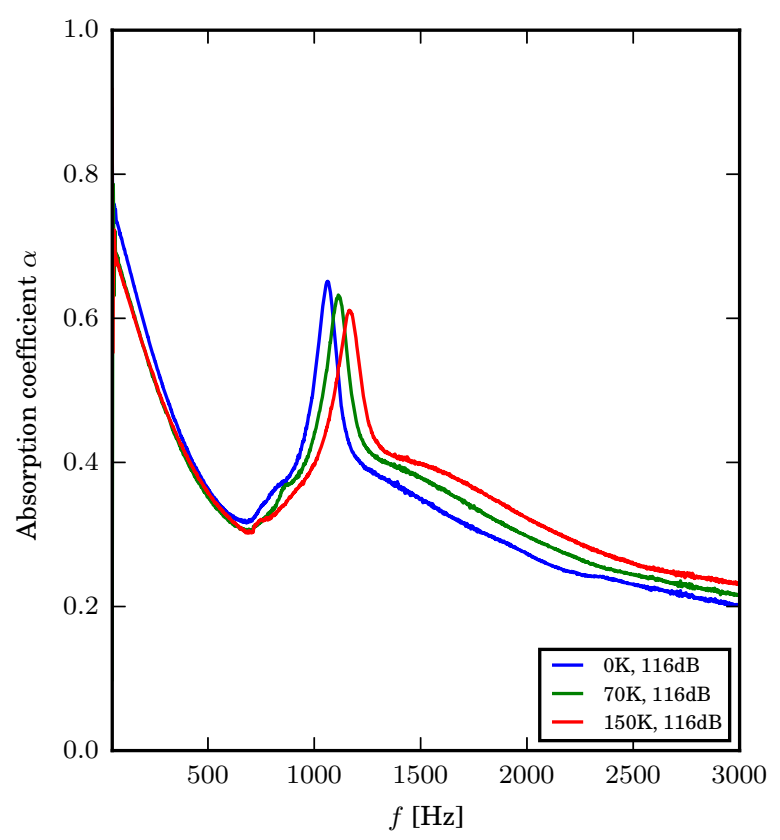

(a) sample \#2

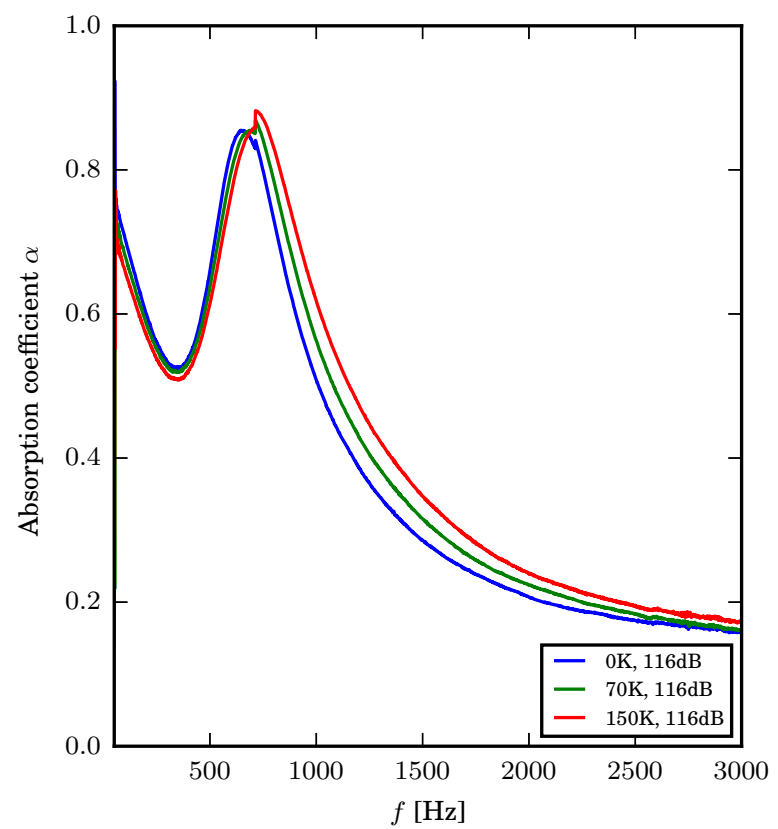

(c) sample \#4

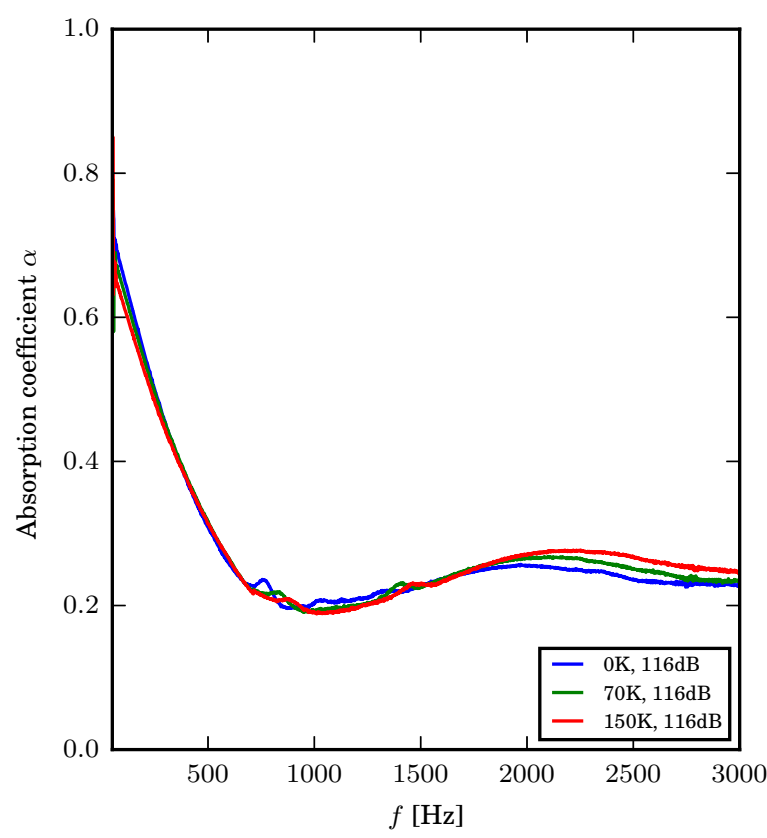

(b) sample \#3

Figure 10 Thermal gradient effect on absorption coefficient for a OASPL=116dB on samples \#2, \#3 and \#4

In this application frequency range, for each sample the effect is slightly the same : a shift in frequency of the maximum value can be observed. Figure 10 shows the effect of the thermal gradient on the absorption coefficient for the other sample. 


\section{B. Impedance model for liner with thermal gradient}

The main objective of this study is to acquire a complete database about temperature gradients effects on acoustic liners impedance. However, a first comparison with existing models can be performed, in order to give some hints for further investigations, even if this is not a major objective of the present study. This subsection will give some comparisons with models in order to take into account the effect of the thermal gradient on the acoustic liner impedance. The liner is composed of a perforated sheet and cavities. In order to decompose the problem, the impedance of the liner can be written as :

$$
Z=Z_{\text {cavity }}+Z_{\text {perforated sheet }},
$$

\section{Impedance of the cavity}

Considering the cavity impedance, the Zinn et al. [27] approach can be applied. The cavities are considered as mono-dimensional cavities with $L$ the cavity length. Assuming a perfect inviscid and non heat conducting gas, let $x$ the axis along the cavity with $x=0$ at the bottom of the cavity and $x=L$ at the open end of the cavity. Expressing each of the variables as the sum of steady and time-dependent small amplitude components :

$$
U=\bar{U}(x)+u(x, t), \quad P=\bar{P}(x)+p(x, t), \quad \rho=\bar{\rho}(x)+\rho(x, t),
$$

the Linearized Euler Equations read :

$$
\begin{aligned}
\frac{\partial u}{\partial t}+\frac{1}{\bar{\rho}} \frac{\partial p}{\partial x} & =0, \\
\frac{\partial p}{\partial t}+\gamma \bar{P} \frac{\partial u}{\partial x} & =0 .
\end{aligned}
$$

Differentiating the momentum equation with respect to $x$ and the energy equation with respect to $t$ and eliminating the cross-derivative term yields the wave equation with variable coefficients :

$$
\frac{\partial^{2} p}{\partial^{2} x}-\frac{1}{\bar{\rho}} \frac{d \bar{\rho}}{d x} \frac{\partial p}{\partial x}-\frac{\bar{\rho}}{\gamma \bar{P}} \frac{\partial^{2} p}{\partial^{2} t}=0 .
$$

Differentiating the steady equation of state and recalling that the steady duct pressure is constant yields the following relationship between the steady temperature and density, i.e. Eq. 25 can be reduced to:

$$
\frac{\partial^{2} p}{\partial^{2} x}-\frac{1}{T} \frac{d T}{d x} \frac{\partial p}{\partial x}-\frac{1}{\gamma R T} \frac{\partial^{2} p}{\partial^{2} t}=0 .
$$

Assuming that the solution has a periodic time dependence (i.e, $\left.p(x, t)=P^{\prime}(x) e^{i \omega t}\right)$ Eq. 26 reduces to the following second order ordinary differential equation for the complex amplitude $P^{\prime}(x)$ :

$$
\frac{\partial^{2} P^{\prime}}{\partial^{2} x}-\frac{1}{T} \frac{d T}{d x} \frac{\partial P^{\prime}}{\partial x}-\frac{\omega^{2}}{\gamma R T} P^{\prime}=0 .
$$

The thermal gradient is considered as linear:

$$
T(x)=T_{0}+m x .
$$

At the bottom of the cavity, $U^{\prime}=0(x=0$, rigid wall). For such a thermal gradient, an analytical solution of Eq. 27 on the acoustic pressure and on the acoustic velocity is available [27]:

$$
\begin{array}{r}
P^{\prime}=a_{1} J_{0}\left(\frac{\omega}{b} \sqrt{T}\right)+a_{2} Y_{0}\left(\frac{\omega}{b} \sqrt{T}\right), \\
U^{\prime}=-\frac{1}{i \omega \bar{\rho}} \frac{d P^{\prime}}{d T} \frac{d T}{d x}=-\frac{m}{|m|} \frac{i}{\bar{\rho} \sqrt{\gamma R T}} a_{1} J_{1}\left(\frac{\omega}{b} \sqrt{T}\right)+a_{2} Y_{1}\left(\frac{\omega}{b} \sqrt{T}\right),
\end{array}
$$

with $a_{1}$ and $a_{2}$ are two complex constants, $J_{n}$ and $Y_{n}$ are respectively $n^{\text {th }}$ order Bessel function and Neumann function, and $b$ is defined as:

$$
b=\frac{|m|}{2} \sqrt{\gamma R}
$$

Finally the impedance of the cavity is:

$$
Z_{\text {cavity }}=-\frac{P^{\prime}(x=L)}{U^{\prime}(x=L)}
$$




\section{Impedance of the perforated sheet}

Concerning the perforated sheet, $\delta$ is the thickness of the sheet, $\phi$ the perforation diameter. The porosity $\sigma$ is defined as the ratio of the perforated surface and the total surface. Let first consider the problem without grazing flow. In Bellucci [28], a semi-empirical model is available :

$$
Z_{\text {perforated sheet }}=i \rho \omega(1 / \sigma)(\delta \Gamma+l) .
$$

$\Gamma$ is defined as :

$$
\Gamma=1-\frac{2 J_{1}\left(k_{s} \phi / 2\right)}{J_{0}\left(k_{s} \phi / 2\right) k_{s} \phi / 2}, \quad k_{s}=\sqrt{-i \omega v}
$$

$l$ is a length correction term which depends on $H e=\frac{\omega \phi}{2 c_{0}}$ and $\sigma$ :

$$
\begin{array}{r}
l=\frac{\phi}{2} \psi(H e) \xi(\sigma), \\
\psi(H e)=2 \cdot 0.8216\left(1+\frac{(0.77 H e)^{2}}{1+0.77 H e}\right)^{-1}, \quad \xi(\sigma)=1-\sqrt{\sigma / 2} .
\end{array}
$$

This model takes into account the viscous effects within the perforation and the end corrections effects. Various models (see [29, 30] for the most classical ones) are similarly constructed. This model does not take into account the SPL effect on the resistance value. Guess [31] proposed an additionnal resistance term $\theta_{N L}$ to take into account the non-linear resistance of an orifice resulting from high sound amplitude:

$$
\theta_{N L}=\frac{\left(1-\sigma^{2}\right)}{\sigma} \frac{\left|u_{0}\right|}{c_{0}}
$$

$\theta_{N L}$ comes from the effect of high sound amplitude and is the result of turbulent dissipation due to vortex formation on the exit sides of the orifices. $\left|u_{0}\right|$ is the amplitude of the acoustic velocity incident to the plate. This term can thus be easily evaluated from the incident sound pressure level on the sample. The factor $\left(1-\sigma^{2}\right)$ can be important for larger $\sigma$. Finally, the considered model for the perforated plate in the impedance tube conditions is :

$$
Z_{\text {perforated sheet }}=i \rho \omega(1 / \sigma)(\delta \Gamma+l)+\theta_{N L} .
$$

Figures 11(a) 11(b) 12(a) and 12(b) show the comparison between the impedance results and the model. The agreement between the resistance model and the measurement is fairly good in the frequency range of interest for industrial application (i.e. around the frequency resonance). The model fails to predict the experimental resistance above $2000 \mathrm{~Hz}$ for most cases. An explanation could be that these semi-empirical models derived from the litterature are usually fitted around the maximum absorption frequency of the considered liners. Regarding the reactance, the model agrees quite well with the experiments for samples \#1, 2 and 3. For sample \#4, the perforated sheet is much thicker so the perforated sheet impedance model is close to its limit of validity, which explains the discrepancies presented in Figure 12(b) 

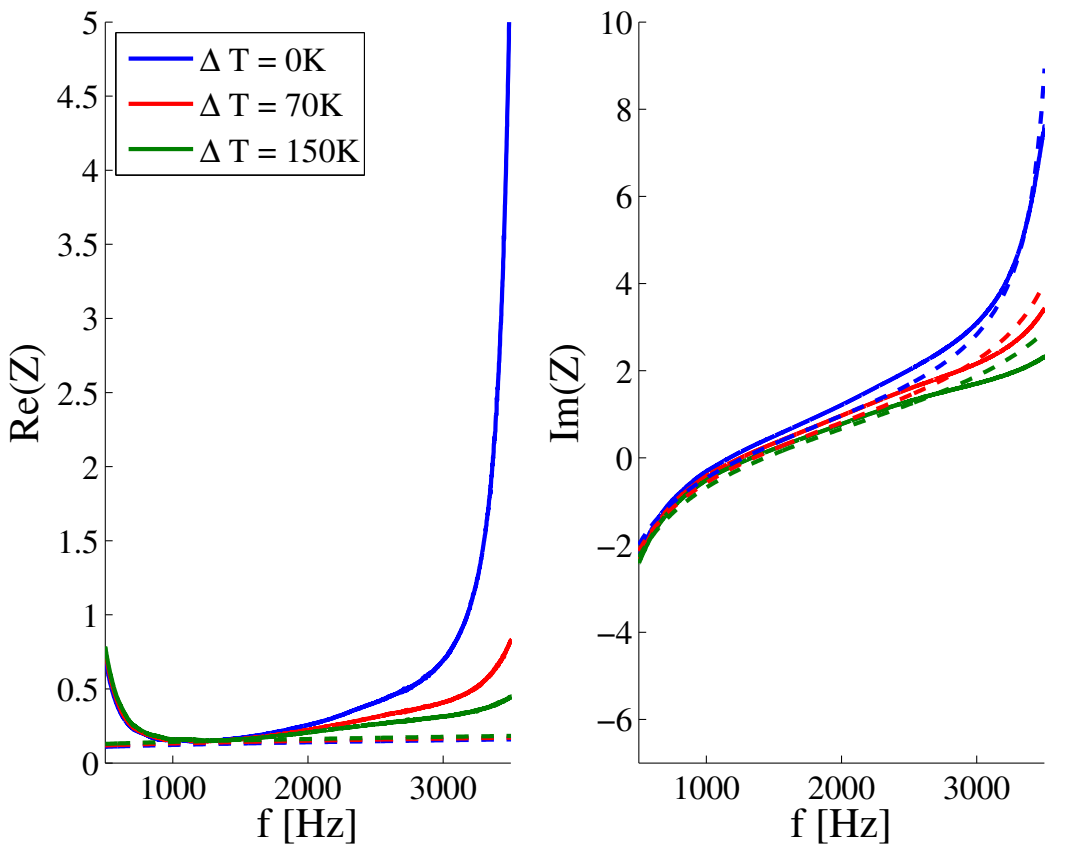

(a) Sample \#1
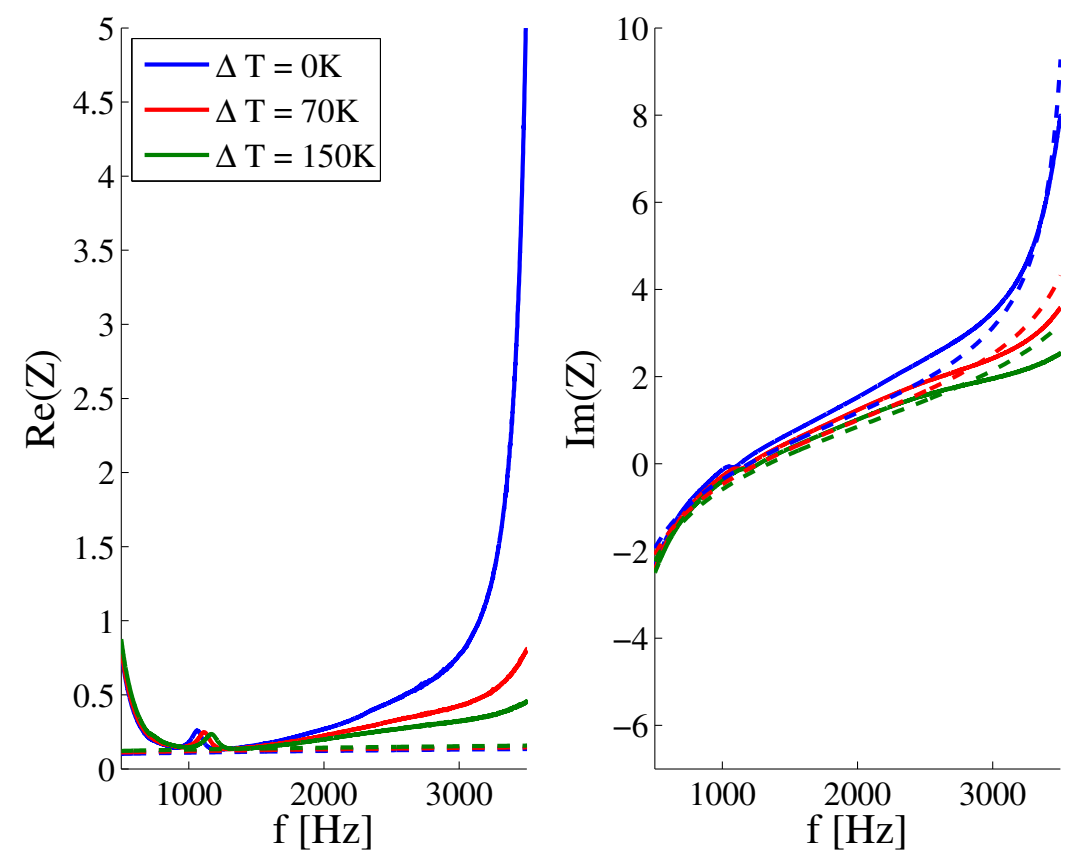

(b) Sample \#2

Figure 11 Impedance measurements (solid line) vs impedance model results (dashed line), OASPL=116dB 

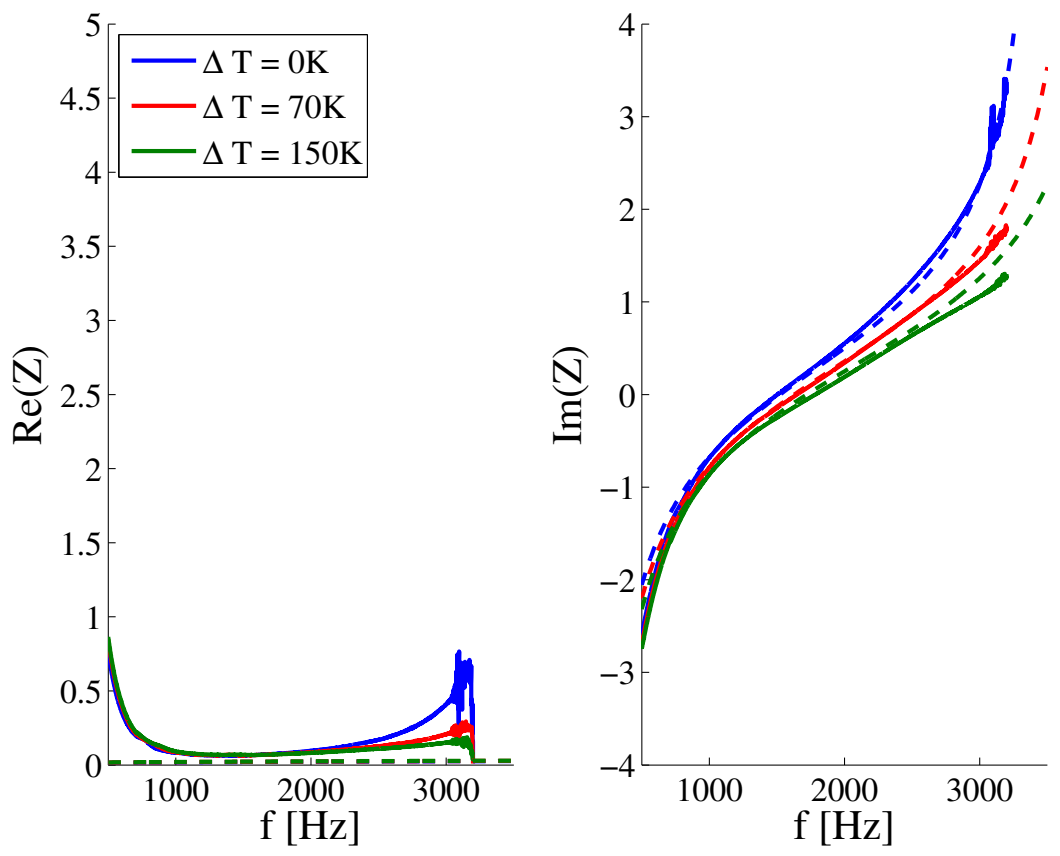

(a) Sample \#3
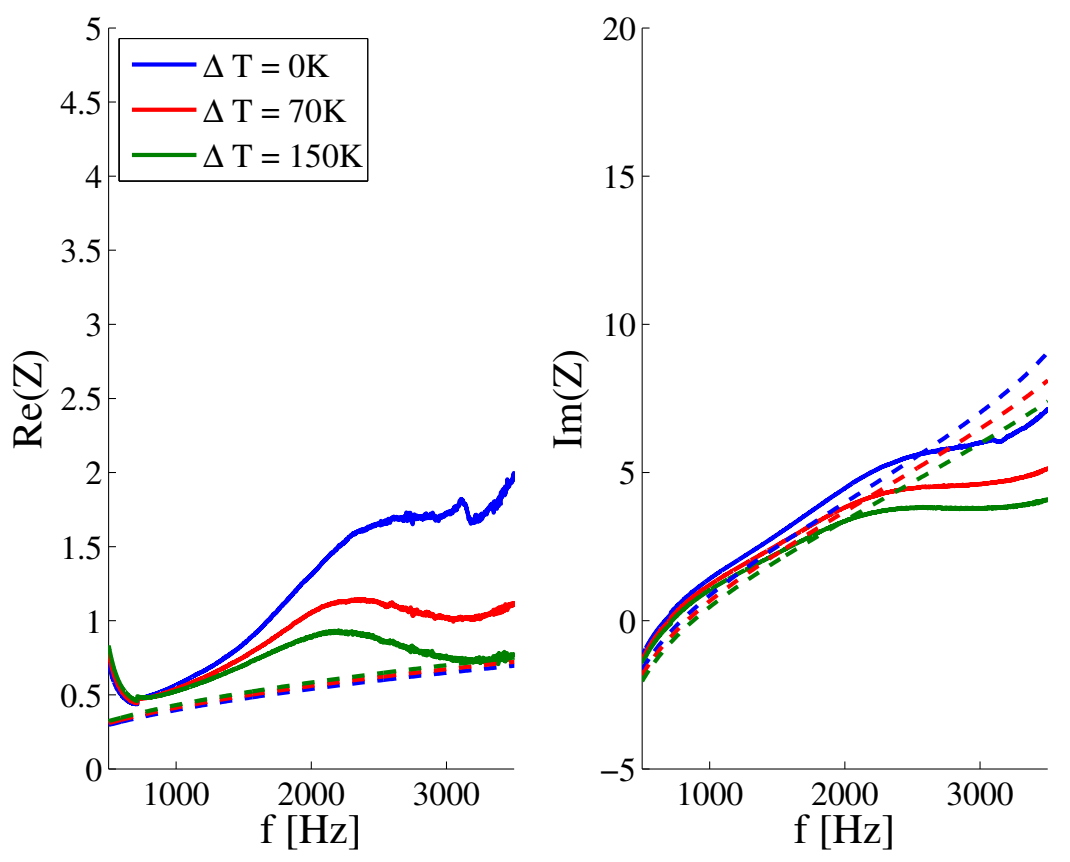

(b) Sample \#4

Figure 12 Impedance measurements (solid line) vs impedance model results (dashed line), OASPL=116dB

\section{Impedance eduction results under grazing flow}

LDA measurement are performed on sample \#3 which was the only sample available for the B2A experiments. Figure 13 presents a typical mean velocity field measured by LDA which is used as an input for the mean flow in the eduction procedure. Figure 14 shows the typical norm of acoustic velocity fields for a given frequency $(f=1240 \mathrm{~Hz})$. For each frequency, the acoustic velocity field is extracted from the LDA measurements and is used as input to the 
eduction problem.

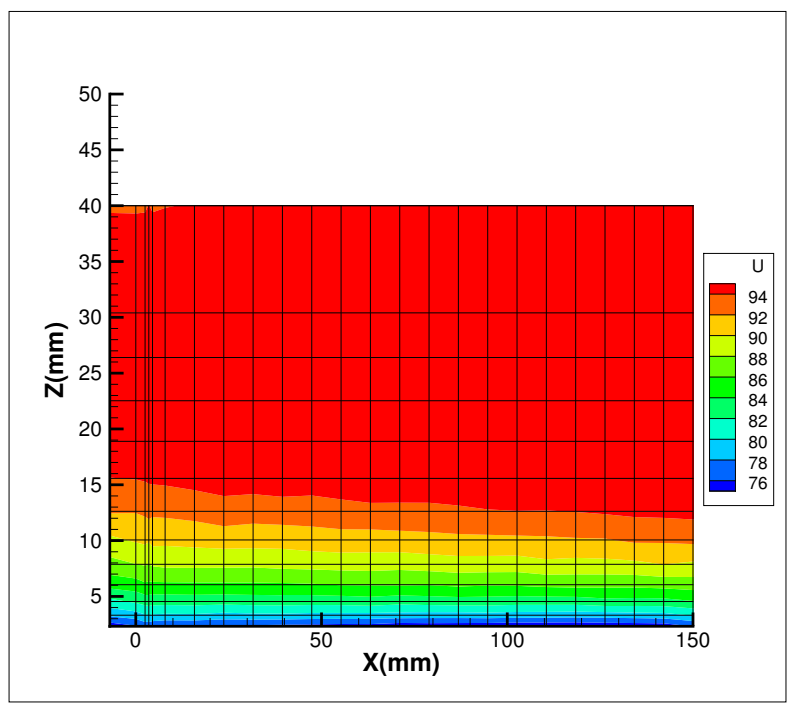

Figure 13 Mean streamwise velocity field $(\mathbf{m} / \mathbf{s})$ measured by LDA, $M_{b}=0.26$

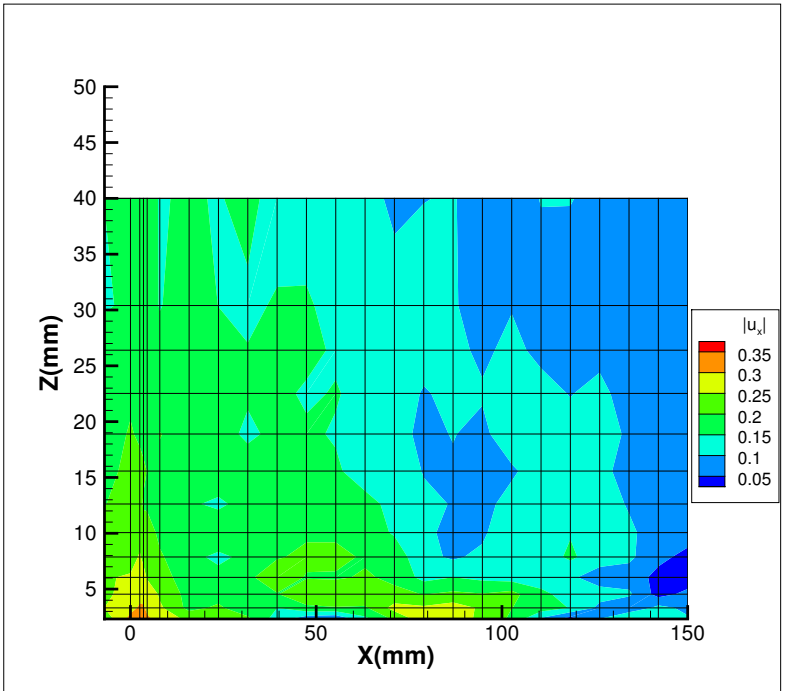

(a) Norm of the streamwise acoustic velocity

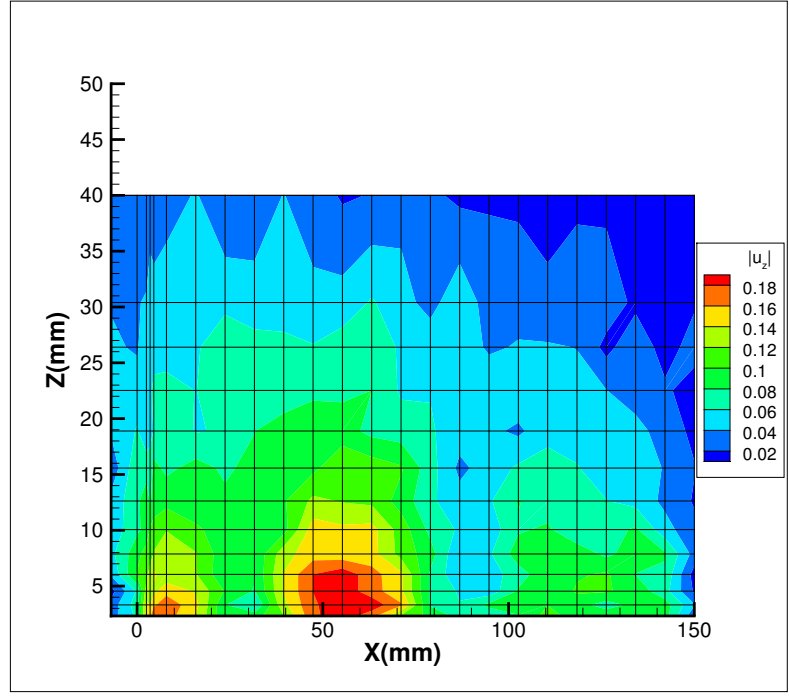

(b) Norm of the normal acoustic velocity

Figure 14 Norm of the acoustic velocities (m/s) extracted from LDA measurements, $f=1240 \mathbf{H z}, M_{b}=0.26$ 


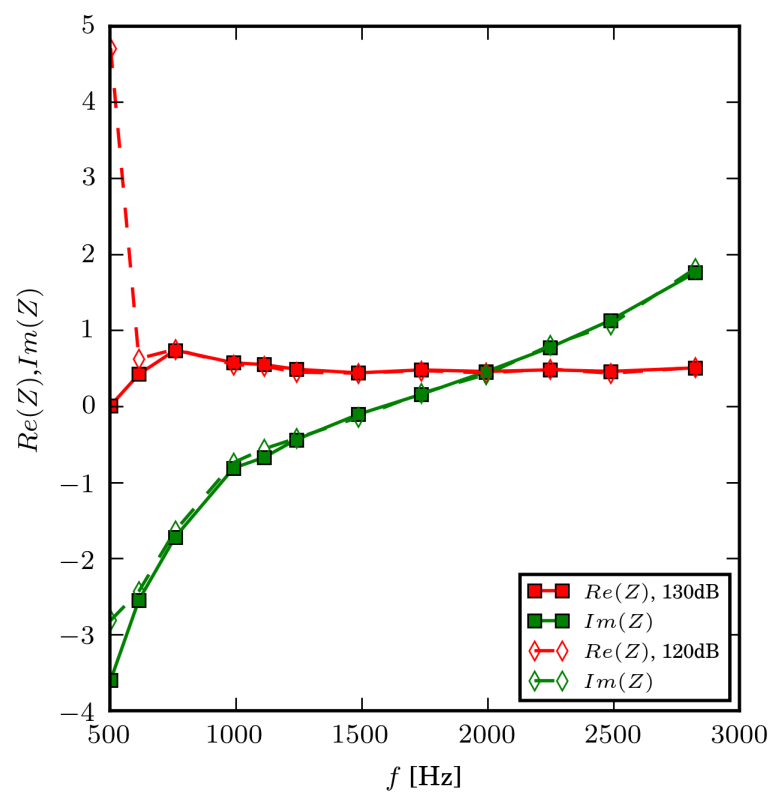

Figure 15 SPL effect for sample \#3 under grazing flow and with a thermal gradient. The bulk Mach number is $M_{b}=0.26$.

Sample \#3 has a non-linear behaviour with the SPL in the impedance tube. The SPL value is here defined as the level of the incident pressure wave of each tone of the multi-sine acoustic source. As this multi-sine signal is made of 12 pure tones, this means that the associated Overall Sound Pressure Level is roughly $10 \mathrm{~dB}$ above the SPL of each tone. Figure 15 shows that in presence of grazing flow, this non-linear behaviour with respect to the SPL is no more highlighted. The resistance of the liner is fully governed by the Mach number over the liner. Therefore, from now on, all the results will be shown for an acoustic source level of $130 \mathrm{~dB}$ per tone. 


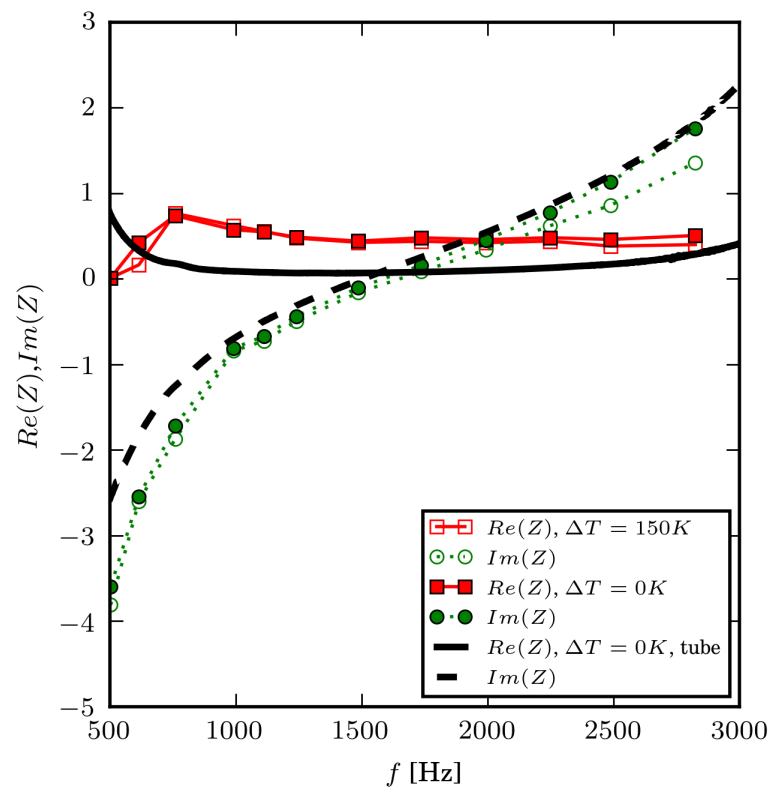

Figure 16 Effect of the temperature gradient for sample \#3 under grazing flow. The bulk Mach number is $M_{b}=0.26$.

Fig 16 shows the effect of the thermal gradient on the liner impedance, in the configuration where the backing plate of the liner is heated. For low frequencies $(f<700 \mathrm{~Hz})$, the eduction method may induce some inaccuracies because the liner absorption is very low. Comparing the tube results and the grazing flow results (B2A) shows that the resistance value is increased with the Mach number. The Mach number implies also a slight decrease of the reactance. The resistance value is mainly not affected by the temperature : only a very little increase can be observed for high frequencies. This means that for the present liner, any temperature influence on the resistance is masked by the resistance increase due to the grazing flow. Though, the reactance value is slightly modified by the thermal gradient which is in line with the model presented in the previous section and the impedance tube results. These reactance variations are mainly due to the change of sound speed within the cavity induced by the thermal gradient.

In order to evaluate roughly the possibility of modeling the liner impedance under thermal gradient, a first attempt is presented here. The impedance under grazing flow can be fully modeled combining the cavity impedance under thermal gradient given by Eq. 32 and a perforated sheet model under grazing flow. In order to take into account of the grazing flow, the Kirby\& Cummings model [32] will be applied. It has to take into account also the friction velocity $v^{*}$, the sound velocity $c_{0}$ of the grazing flow. $v$ is the cinematic viscosity. The impedance of the perforated sheet reads :

$$
Z_{\text {perforated sheet }}=R+i X,
$$

with

$$
\begin{array}{r}
R=\frac{\sqrt{8 v \omega}}{\sigma \cdot c_{0}} \frac{\delta}{\phi}+\frac{\left(26.16\left(\frac{\delta}{\phi}\right)^{-0.169}-20\right) v^{*}}{\sigma \cdot c_{0}}-\frac{4.055 f \phi}{\sigma \cdot c_{0}}, \\
X=\frac{\omega}{\sigma \cdot c_{0}}\left(\delta+\epsilon \frac{8 \phi}{3 \pi}\right), \\
\epsilon=\min \left[1,\left(1+0.6 \frac{\delta}{\phi}\right) \exp -\frac{\frac{v^{*}}{f \delta}-0.18 \frac{\phi}{\delta}}{1.8+\frac{\delta}{\phi}}-0.6 \frac{\delta}{\phi}\right] .
\end{array}
$$

Figure 17(a) presents the comparison between the impedance model made by the sum of Eq 32 and Eq 39 and the educed results under grazing flow and with a classical thermal gradient of $150 \mathrm{~K}$. The agreement is quite good. Kirby\& Cummings model does not take into account the SPL effect on the impedance but the main effect on the resistance value 
is the grazing flow effect so the agreement in term of resistance is satisfactory. The simple gradient model for the cavity under thermal gradient is also sufficient in order to correctly reproduce the experimental trend. As the objective of this study is purely experimental, the impedance model could be widely improved and this will be an interesting perspective. Fig 17(b) is an illustration of the model/experiments comparison for the configuration without thermal gradient. The agreement is also very good.

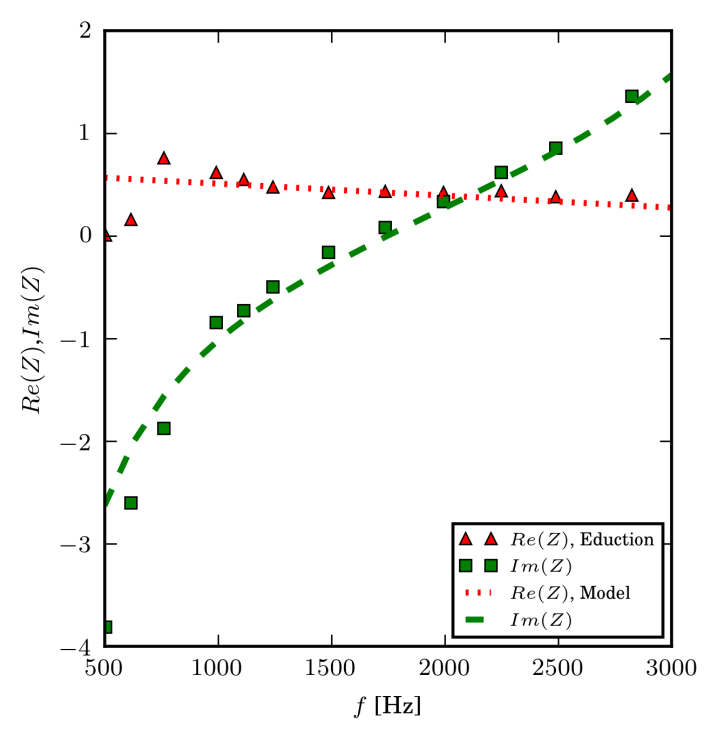

(a) $\Delta T=150 K$

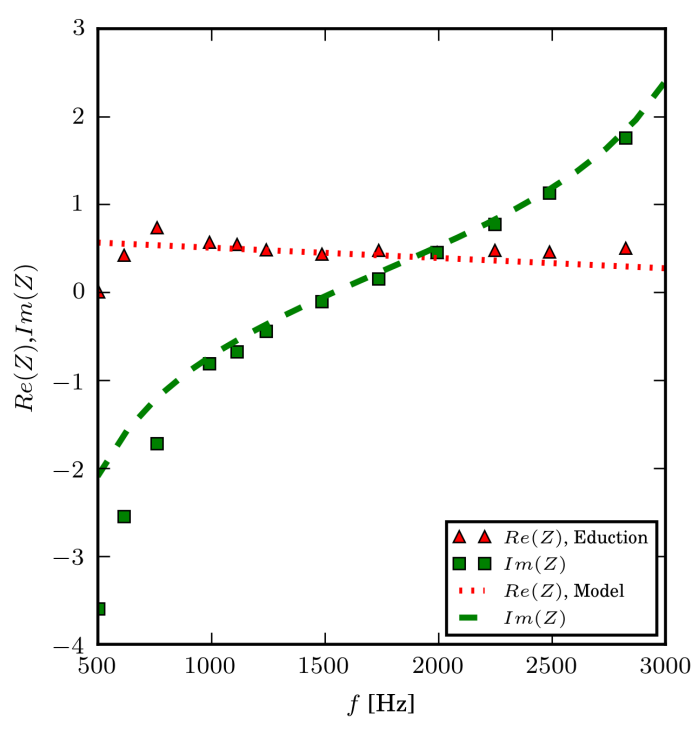

(b) $\Delta T=0 K$

Figure 17 Impedance measurements (square) vs impedance model results (dashed line) for sample \#3, $M_{b}=$ 0.26

\section{Effect of acoustics on the thermal gradient}

Figures 18(a) and 18(b) present the effect of acoustics on the temperature at the perforated sheet for high SPL in the impedance tube configuration. No effect is noticed for lower SPL level. The temperature is clearly modified (decrease from 7 to $10 \mathrm{~K}$ ) with high SPL [8]. Regarding the Biot number, for a $70 \mathrm{~K}$ thermal gradient the Biot number is 2.27 and for a $150 \mathrm{~K}$ thermal gradient the Biot number is 2.02 . It is increased probably because the acoustics forcing of the flow modifies the value of the convection heat transfer coefficient $h$. The convection heat transfer coefficient is increased because of forced convection due to the acoustic excitation. When the liners are subjected to high excitation levels, the acoustic velocity fluctuation in the near-wall region $w$ is no more a purely sinusoidal fluctuation with a zero mean value. This has been particularly emphasized in the literature using Direct Numerical Simulations carried out on this type of configuration. This is explained by the generation, during a half-period of the excitation cycle, of a vortex above the upper orifice of the hole, whereas during the other half-period a similar mechanism takes place inside the cavity. These phenomena are explained numerically for instance in the work of Zhang \& Bodony [33] and experimentally exhibited in Ingard \& Labate [34] or Hersh \& Walker [35]. We can infer that this modification of the near-wall region $w$ increases the $h$ convection heat transfer coefficient. 


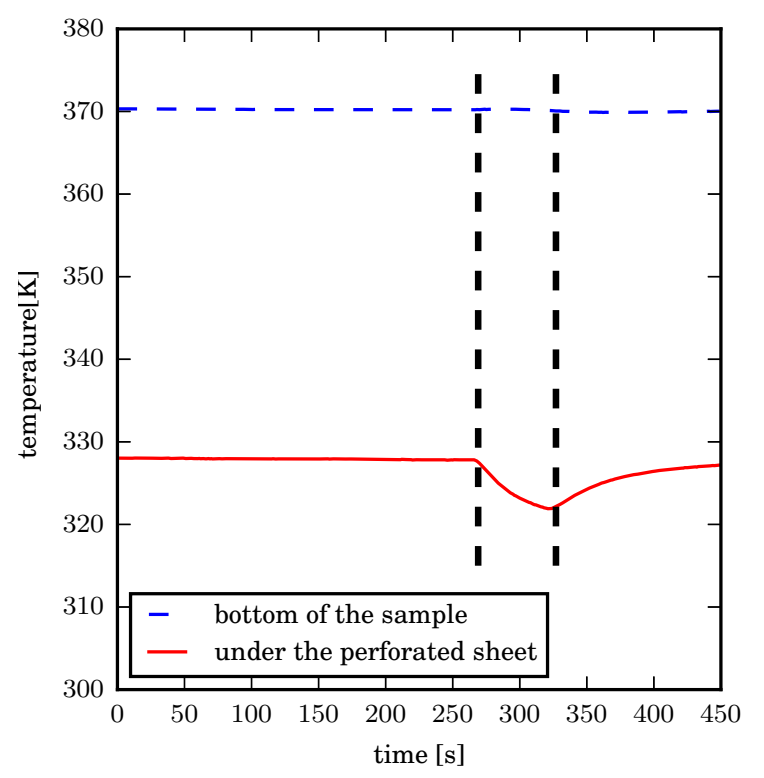

(a) $\Delta T=70 K$

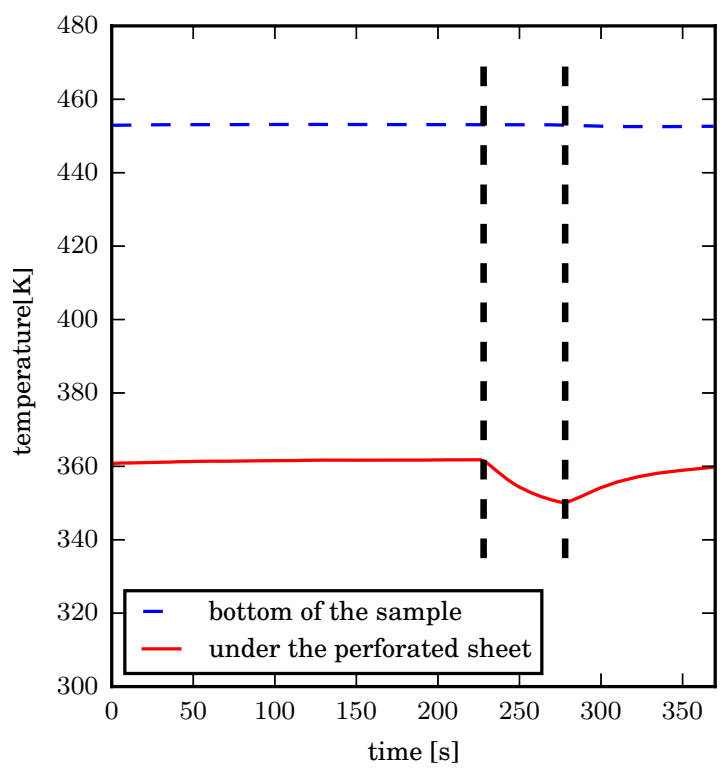

(b) $\Delta T=150 K$

Figure 18 Effect of the acoustic on the temperature gradient. Dashed vertical lines : the beginning and the stop of the white noise excitation (146dB OASPL)

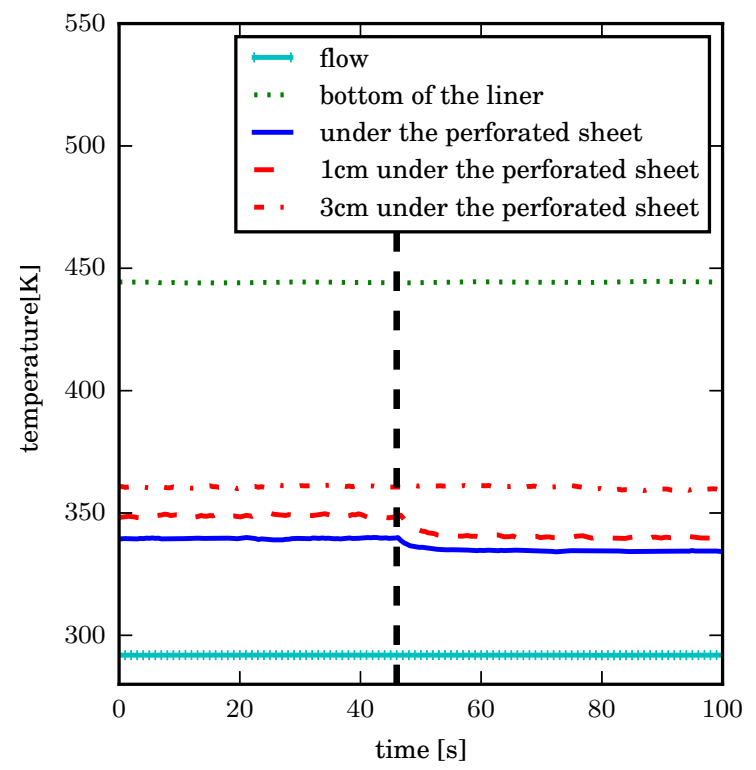

Figure 19 Effect of the acoustic excitation on the temperature in the liner cells during a run. Dashed vertical line : acoustic (140dB OASPL) excitation switched on.

The same phenomenon can be observed in the B2A configuration with grazing flow [36]. Fig 19 represents the effect of acoustics at several positions below the perforated sheet. In a same way as for the impedance tube case, the $h$ convection heat transfer coefficient seems to be increased. This increase occurs very close below the perforated sheet, it is a very local phenomenon. 


\section{Conclusions}

This study assessed the temperature effect on the impedance of conventional SDOF liners, both without and with grazing flow and both in the linear and nonlinear regime with respect of the acoustic sound pressure level. For most of the study the surface of the liner is kept at a temperature lower than the backing of the cavity, with a temperature gradient up to $150 \mathrm{~K}$. One test was also made with a sample heated at the top, through heated devices included within the perforated sheet. Experiments are performed in a controlled environment, with a detailed monitoring of the temperature all along the liner sample. The liner impedance is either derived from the reflection coefficient measured in a normal impedance tube, or is educed with an inverse method from acoustic velocity or wall pressure fields measured in the ONERA grazing flow duct (B2A). A complete database is now available in order to understand the influence of the thermal gradient on the impedance and the acoustic source level on the temperature of the sample. The impact of thermal gradient on the acoustic impedance (with and without grazing flow) seems only to be a change on the reactance (shift of the anti-resonance frequency). However, the acoustics seem to directly impact the temperature repartition inside the cavity for high Sound Pressure Level. Further modeling studies will enable to understand this particular coupling between acoustics and the thermal gradient within the liner

\section{Acknowledgments}

This work has been funded within the frame of the Joint Technology Initiative JTI Clean Sky 2, AIRFRAME Integrated Technology Demonstrator platform "AIRFRAME ITD" (contract CSJU-CS2-GAM-AIR-2014-15-01 Annex 1, Issue B04, October 2nd, 2015) being part of the Horizon 2020 research and Innovation framework programme of the European Commission. Gillian Leplat and Thomas Batmalle's help is gratefully acknowledged for the thermal conductivity measurements.

\section{References}

[1] Foster, P., “Airflow passenger liner,”, 2010. US Patent 12897270.

[2] Rogero, J., Roux, A., Lempereur, P., and Rechain, B., "Paroi acoustique à échangeur thermique intégré,”, 2016. EP 3038101A1.

[3] Baccouche, R., Moreau, S., and Tahar, M. B., "Test of single degree of freedom acoustic treatment impedance models for multimodal acoustic propagation in duct with flow," Journal of Acoustical Society of America, Vol. 141, No. 6, 2017, pp. 4168-4178. doi:10.1121/1.4983653.

[4] Temiz, M. A., Tournadre, J., Arteaga, I. L., and Hirschberg, A., "Non-linear acoustic transfer impedance of micro-perforated plates with circular orifices," Journal of Sound and Vibration, Vol. 366, 2016, pp. 418-428. doi:10.1016/j.jsv.2015.12.022.

[5] Lahiri, C., and Bake, F., "A review of bias flow liners for acoustic damping in gas turbine combustors," Journal of Sound and Vibration, Vol. 400, 2017, pp. 564-605. doi:10.1016/j.jsv.2017.04.005.

[6] Acker, T., and Mitchell, C., "Combustion zone - Acoustic cavity interactions in rocket combustors," Journal of Propulsion and Power, Vol. 10, 1994, pp. 235-243. doi:10.2514/3.23734.

[7] Laudien, E., Pongratz, R., Pierro, R., and Preclik, D., "Experimental Procedures Aiding the Design of Acoustic Cavities," Liquid Rocket Engine Combustion Instability, edited by V. Yang and W. Anderson, AIAA, Washington DC, 1995, pp. 377-399.

[8] Bies, D., and Wilson, O., "Acoustic Impedance of a Helmholtz Resonator at Very High Amplitude," Journal of Acoustical Society of America, Vol. 29(6), 1957, pp. 711-714. doi:10.1121/1.1909021.

[9] Lahiri, C., Knobloch, K., Bake, F., and Enghardt, L., "Acoustic Measurements of Perforated Liners in Hot and Pressurized Flow," ASME Turbo Expo 2013: Turbine Technical Conference and Exposition, GT2013-94674, San Antonio, USA, 2013. doi:10.1115/GT2013-94674.

[10] Burd, D., and Eversman, W., "Normal Incidence Impedance Measurement At Elevated Temperatures," 17th AIAA/CEAS Aeroacoustics Conference, AIAA 2017-3025, Portland, USA, 2011. doi:10.2514/6.2011-2931.

[11] Elnady, T., Bodén, H., and Kontio, T., "Impedance of SDOF perforated liners at high temperatures," 10th AIAA/CEAS Aeroacoustics Conference, Manchester, UK, 2004. doi:10.2514/6.2004-2842.

[12] Kabral, R., Boden, H., and Elnady, T., "Determination of Liner Impedance under High Temperature and Grazing Flow Conditions," 20th AIAA/CEAS Aeroacoustics Conference, AIAA 2014-2956, Atlanta, USA, 2014. doi:10.2514/6.2014-2956. 
[13] Rademaker, E., Wal, H. V. D., and Geurts, E., "Hot-stream in-situ acoustic impedance measurements on various air-filled cavity and porous liners," 16th International Congress on Sound and Vibration, ICSV16, Krakow, Poland, 2009.

[14] Bothien, M. R., and Wassmer, D., "Impact of Density Discontinuities on the Resonance Frequency of Helmholtz Resonators," AIAA Journal, Vol. 53, No. 4, 2015. doi:10.2514/1.J053227.

[15] Minotti, A., Simon, F., and Gantié, F., "Characterization of an acoustic liner by means of Laser Doppler Velocimetry in a subsonic flow," Aerospace Science and Technology, 2007. doi:10.1016/j.ast.2007.09.007.

[16] Reulet, P., and Leplat, G., "An Inverse Method for Experimental Determination of Temperature Dependent Thermal Conductivities and Specific Heat of Orthotropic Materials," ITCC 2011 - International Thermal Conductivity Conference, Chicoutimi, Quebec, CANADA, 2011.

[17] Colburn, A., “A method of correlating forced convection heat transfer,” Trans. AIChE, Vol. 29, 1933, pp. 174-210.

[18] Chung, J. Y., and Blaser, D. A., "Transfer function method of measuring in-duct acoustic properties. I. Theory," J. Acoust. Soc. Am., Vol. 68, No. 3, 1980, pp. 907-913. doi:10.1121/1.384778.

[19] Boden, H., and Abom, M., "Influence of errors on the two microphones method for measuring acoustics properties in ducts," Journal of the Acoustic Society of America, Vol. 79, No. 2, 1986, pp. 541-549. doi:10.1121/1.393542.

[20] Leon, O., Piot, E., Sebbane, D., and Simon, F., "Measurement of acoustic velocity components in a turbulent flow using LDV and high-repetition-rate PIV," Experiments in fluids, 2017. doi:10.1007/s00348-017-2348-4.

[21] Primus, J., Piot, E., and Simon, F., "An adjoint-based method for liner impedance eduction: Validation and numerical investigation," Journal of Sound and Vibration, Vol. 332, No. 1, 2013, pp. 58-75. doi:10.1016/j.jsv.2012.07.051.

[22] Minotti, A., Simon, F., and Gantié, F., "Characterization of an acoustic liner by means of Laser Doppler Velocimetry in a subsonic flow,", 2008. doi:10.1016/j.ast.2007.09.007.

[23] Lavieille, M., Simon, F., and Micheli, F., "Measurement of acoustic quantity fields in duct flow by Laser Doppler Velocimetry." Proceedings of the 12th AIAA/CEAS Aeroacoustics Conference, AIAA-2006-2550, Cambridge, Massachusetts, 2006.

[24] Pascal, L., Piot, E., and Casalis, G., "Discontinuous Galerkin Method for acoustic modes computation in lined ducts," 18th AIAA/CEAS Aeroacoustics conference, AIAA 2012-2153, Colorado Springs, Colorado, USA, 2012.

[25] Piot, E., Primus, J., and Simon, F., "Liner impedance eduction technique based on velocity fields," 18th AIAA/CEAS Aeroacoustics Conference, AIAA 2012-2198, Colorado Springs, Colorado, USA, 2012. doi:10.2514/6.2012-2198.

[26] Byrd, R. H., Nocedal, J., and Schnabel, R. B., "A limited memory algorithm for bound constrained optimization," SIAM Journal on Scientific and Statistical Computing, Vol. 16, 1995, pp. 1190-1208.

[27] Sujith, R. I., Waldherr, G. A., and Zinn, B. T., "An exact solution for one-dimentional acoustic fields in ducts with an axial temperature gradient," Journal of Sound and Vibration, Vol. 184, 1995, pp. 389-402. doi:10.1006/jsvi.1995.0323.

[28] Bellucci, V., Flohr, P., and Paschereit, C. O., "Numerical and experimental study of acoustic damping generated by perforated screens," AIAA Journal, Vol. 42, No. 8, 2004, pp. 1543-1549.

[29] Melling, T. H., "The acoustic impedance of perforates at medium and high sound pressure levels," Journal of Sound and Vibration, Vol. 29, No. 1, 1973, pp. 1-65. doi:10.1016/S0022-460X(73)80125-7.

[30] Cummings, A., "Acoustic nonlinearities and power losses at orifices," AIAA Journal, Vol. 22, 1984, pp. 786-792. doi: $10.2514 / 3.8680$.

[31] Guess, A., "Calculation of perforated plate liner parameters from specified acoustic resistance and reactance," Journal of Sound and Vibration, Vol. 40(1), 1975, pp. 119-137. doi:10.1016/S0022-460X(75)80234-3.

[32] Kirby, R., and Cummings, A., "The impedance of perforated plates subjected to grazing gas flow and backed by porous media," Journal of Sound and Vibration, Vol. 217, 1998, pp. 619-636. doi:10.1006/jsvi.1998.1811.

[33] Zhang, Q., and Boden, D., "Numerical investigation of a honeycomb liner grazed by laminar and turbulent boundary layers," Journal of Fluid Mechanics, Vol. 792, 2016, pp. 936-980. doi:10.1017/jfm.2016.79.

[34] Ingard, U., and Labate, S., "Acoustic circulation Effects and the Non linear Impedance Orifices," Journal of the Acoustical Society of America, Vol. 22(2), 1950, pp. 211-218. doi:10.1121/1.1906591. 
[35] Hersh, A. S., and Walker, B., "The Acoustic Behavior of Helmholtz Resonators Exposed to High Speed Grazing Flows," AIAA 3rd AeroAcoustics Conference, 1976.

[36] Mery, F., Conte, C., Leon, O., and Piot, E., "Non-intrusive measurements of velocity fields over a lined wall in a turbulent duct flow for drag assessment," 23rd AIAA/CEAS Aeroacoustics Conference, AIAA 2017-3025, Denver, USA, 2017. doi: 10.2514/6.2017-3025. 